\title{
Impact of Interactions Between Self-Reported Psychological Stress and Habitual Exercise on the Dietary Intake of Japanese Men and Women: a Large-Scale Cross-Sectional Study
}

\author{
Kaori Endoh $^{1}$, Kiyonori Kuriki ${ }^{1 *}$, Nobuhiko Kasezawa ${ }^{2}$, Kazushige Tohyama ${ }^{2}$, \\ Toshinao Goda ${ }^{3}$
}

\begin{abstract}
Background: Modifying lifestyle factors such as diet and exercise can reduce the risk of cancer. Psychological stress (PS) might be indirectly associated with cancer because it alters lifestyle factors. However, the relationship among these variables has not been fully investigated. Thus, we examined interactions between self-reported PS (SRPS) and habitual exercise on diet. Materials and Methods: In all, 5,587 men and 2,718 women were divided into "exerciser" and "non-exerciser" groups, based on whether they exercised regularly, and classified into three SRPS levels: low, moderate and high. Diet was estimated using a validated food frequency questionnaire. Using a general linear model, food and nutrient consumption was estimated for each SRPS level in the 2 exercise groups, and the interactions between SRPS levels and exercise were calculated. Results: In women, the intake of pork and beef, low fat milk and yogurt, natto (fermented soybean), carrots and squash, other root vegetables, mushrooms, seaweeds, and wine, along with the nutrients vegetable protein, soluble, insoluble and total dietary fiber, daidzein, genistein, carotene, retinol equivalents, vitamin B2, pantothenic acid, potassium, calcium, magnesium, phosphorus and iron demonstrated significant interaction with SRPS and habitual exercise (p for interaction $<0.05$ ). In men, raw and green leafy vegetables and fruit and vegetable juice significantly interacted with SRPS and habitual exercise ( $p$ for interaction $<\mathbf{0 . 0 5}$ ). Conclusions: We suggest that certain foods and nutrients, which are thought to have a protective effect against cancer, interact with SRPS and habitual exercise, especially in women. This information is valuable for understanding and improving interventions for cancer prevention.
\end{abstract}

Keywords: Cancer prevention - dietary intake - exercise - psychological stress - Japan

Asian Pac J Cancer Prev, 17 (4), 2007-2017

\section{Introduction}

In 2012, cancer was the leading cause of death in the world; 8.2 million people died from cancer worldwide (World Health Organization, 2015). It is estimated that $30 \%$ of deaths from cancer are associated with adult obesity and diet and 5\% with a sedentary lifestyle (Harvard Center for Cancer Prevention, 1996). Furthermore, studies have found that vegetables and their related vitamins reduce the risk of various types of cancer (World Cancer Research Fund American Institute for Cancer Research, 2007). Convincing evidence exists showing that exercise decreases the risk of colon cancer, and some evidence exists suggesting that exercise decreases the risk of breast and endometrial cancer as well (World Cancer Research Fund American Institute for Cancer Research, 2007). In Japan, cancer has been the leading cause of death since 1981. To decrease deaths from cancer, the Ministry of Labour and Welfare in Japan announced a new plan for cancer in December of 2015, including an increased focus on the research, prevention, and treatment of cancer. Thus, research on cancer prevention in Japanese populations is needed, particularly research related to lifestyle factors such as diet and physical activity.

Cancer leads to psychological stress (PS) in patients (Massie, 2004). However, the association between PS and cancer is controversial. Some studies found that PS does not cause cancer (Schernhammer et al., 2004; Santos et al., 2009). Other studies found a positive association between PS and cancer (Jansson et al., 2009). It is possible that PS influences the risk of cancer indirectly (Chida et al., 2008), because PS is associated with lifestyle factors that are related to cancer risk, such as diet (Laugero et al., 2011) and exercise (Perales et al., 2014). Heikkila et al reported that job stress was a risk factor for an unhealthy lifestyle, including a high BMI, smoking, drinking, and lack of exercise (Heikkila et al., 2013). In our previous study, an unhealthy lifestyle was based on drinking, smoking, and 
exercise (Endoh et al., 2015b). Significant differences in food intake were observed across the three levels of SRPS for participants with an unhealthy lifestyle (chi-squared test; $\mathrm{p}<0.001$ for men and women). However, few studies have examined the specific associations between PS, habitual exercise, and dietary intake of food and nutrients. In the present study, we investigated the interaction between self-reported PS (SRPS) and habitual exercise on the dietary intake of various foods and nutrients in Japanese men and women.

\section{Materials and Methods}

\section{Subjects}

From July 2005 to March 2007, we recruited individuals who attended a health checkup at a local health center in Shizuoka, Japan (Mochizuki et al., 2011; Endoh et al., 2015a; Endoh et al., 2015b). An in-person explanation was provided and written informed consent for participation in the study was obtained from 12,066 subjects. Of these, 3,761 (31.2\%) met the following exclusion criteria: (1) less than 29 or greater than 79 years of age ( $n=84,0.7 \%)$, (2) missing value for SRPS $(n=663,5.5 \%),(3) 61$ years of age or greater $(n=2,373$, $19.7 \%$ ), excluded because most were retired and had no job-related stress, (4) past or present history of depression or being treated with an anti-depressant or tranquilizer $(n=189,1.6 \%),(5)$ energy intake $(n=793,6.6 \%)$ and body mass index $(\mathrm{BMI})(\mathrm{n}=761,6.3 \%)$ greater or less than 2 standard deviations from the mean, (6) missing data for lifestyle factors $(n=489,4.1 \%),(7)$ extremely short or long sleep times $(n=246,2.0 \%)$, and (8) past or present disease history of stroke, myocardial infarction, or cancer $(n=442$, $3.7 \%)$. Ultimately, 5,587 (46.3\%) men and 2,718 (22.5\%) women were included in the analysis. This study was conducted according to the Declaration of Helsinki and all procedures involving human subjects were approved by the Ethics Committee of the University of Shizuoka.

\section{Lifestyle assessment}

Anthropometric measurements such as height, weight, and blood pressure were taken by trained staff at a local health center. Blood was taken after an overnight fast, and $\mathrm{HbA1c}$, total cholesterol, high-density lipoprotein cholesterol, and triglycerides were measured. Information on age, smoking status (never, past, or current), sleeping time (hours per day), SRPS, and present and past disease history was obtained by trained medical staff. To calculate BMI, weight ( $\mathrm{kg}$ ) was divided by height $(\mathrm{m})$ squared $(\mathrm{kg} /$ $\mathrm{m}^{2}$ ). To determine SRPS, we asked each participant "Do you feel stress?". The four possible answers (no, little, moderate, or extreme) were classified as follows: low ( $n=206$ responded "no" and $n=1,993$ "little"), moderate $(n=4,938$ responded "moderate") and high $(n=1,168$ responded "extreme"). To assess habitual exercise, the subjects were asked "Do you exercise regularly?" and answered either yes or no. The subjects who answered yes were considered exercisers and those who answered no were non-exercisers. A scientifically validated food frequency questionnaire (Kobayashi et al., 2011; Kobayashi et al., 2012) was used. This questionnaire contains 58 food items and foods, which were used to calculate the intake of energy and 42 nutrients. The dietary intake of each food and nutrient was calculated and adjusted for energy intake.

\section{Statistical analyses}

The adjusted means for the dietary intake of food and nutrients were calculated by stratifying participants by the presence or absence of habitual exercise (i.e., the exerciser and non-exerciser groups). A general linear model was used to assess the significant interactions between SRPS levels and habitual exercise on the dietary intake of food and nutrients. The model was adjusted for the following confounding variables, as in our previous studies (Endoh et al., 2015a; Endoh et al., 2015b): age (years), BMI $\left(\mathrm{kg} / \mathrm{m}^{2}\right)$, dietary energy ( $\mathrm{kcal} /$ day), alcohol intake (g/day), sleeping time (hours/day), smoking status (never, past, or current smoker), and past or present history of hypertension and diabetes mellitus (yes or no). All reported $p$ values were based on two-sided tests. Statistical analyses were conducted using PASW Statistics 18 (SPSS Inc.), and $\mathrm{p}<0.05$ was considered statistically significant.

\section{Results}

Table 1 shows the demographic characteristics of the subjects. The mean BMI and percentages of participants who drink and smoke were higher in men. The proportions of participants with each SRPS level and who exercise

Table 1. Subject Characteristics

\begin{tabular}{lcc}
\hline \multicolumn{1}{l}{} & Men & Women \\
\hline Number & 5,587 & 2,718 \\
Age (years) & $48.1 \pm 7.4$ & $46.4 \pm 7.7$ \\
BMI (kg/m $\left.{ }^{2}\right)$ & $23.5 \pm 2.5$ & $21.3 \pm 2.4$ \\
SRPS (\%) & & \\
Low & 27.9 & 23.6 \\
Moderate & 58.1 & 62.2 \\
High & 14.0 & 14.2 \\
Habitual exercise (\%) & & \\
Exercisers & 52.2 & 54.6 \\
Non-exercisers & 47.8 & 45.4 \\
Drinking habit (\%) & & \\
Non-drinkers & 27.1 & 65.0 \\
Drinkers & 72.9 & 35.0 \\
Smoking habit (\%) & & \\
Never & 37.7 & 87.5 \\
Past & 21.1 & 4.0 \\
Current & 41.2 & 8.5 \\
HbAlc (\%) & $5.6 \pm 0.8$ & $5.3 \pm 0.9$ \\
Serum TC (mmol/L) & $5.5 \pm 0.9$ & $5.5 \pm 0.9$ \\
Serum HDL-C (mmol/L) & $1.5 \pm 0.4$ & $1.9 \pm 0.4$ \\
Serum TG (mmol/L) & $1.6 \pm 1.2$ & $0.9 \pm 0.5$ \\
Sleeping time (hours/day) & $6.4 \pm 0.8$ & $6.3 \pm 0.9$ \\
History of diabetes mellitus (current or previous) & $(\%)$ \\
Yes & 4.0 & 0.9 \\
No & 96.0 & 99.1 \\
History of hypertension (current or previous) (\%) & \\
Yes & 10.2 & 4.5 \\
No & 89.8 & 95.5 \\
\hline Do & & \\
\hline
\end{tabular}

Data are presented as the mean \pm SD; SRPS, Self-reported psychological stress; BMI, body mass index; TC, total cholesterol; HDL-C, highdensity lipoprotein cholesterol; TG, triglycerides. 
Impact of Interaction of Psychological Stress with Habitual Exercise on Dietary Intake

were approximately equal between men and women.

Table 2 shows the interactions between SRPS levels and habitual exercise for the dietary intake of foods among middle-aged Japanese men and women. In male non-exercisers, SRPS was positively associated with the dietary intake of "cakes, cookies, and biscuits" and wine and negatively associated with dietary intake of potatoes, "tomatoes, tomato ketchup, boiled tomatoes, and stewed tomatoes", seaweeds, and beer ( $\mathrm{p}$ for trend $<0.05$ for all). In male exercisers, SRPS was positively associated with

Table 2. Interactions between SRPS and Habitual Exercise Level in the Dietary Intake of Food in Middle-Aged Japanese Men And Women ${ }^{1}$

\begin{tabular}{|c|c|c|c|c|c|c|c|c|c|c|}
\hline \multirow{3}{*}{ (g/1000 kcal/day) } & \multicolumn{5}{|c|}{ Men $(n=5,587)$} & \multicolumn{5}{|c|}{ Women $(n=2,718)$} \\
\hline & \multicolumn{3}{|c|}{ SRPS } & \multirow{2}{*}{$\begin{array}{l}\mathrm{p} \text { for } \\
\text { trend }\end{array}$} & \multirow[b]{2}{*}{$\mathrm{p}$ for int } & \multicolumn{3}{|c|}{ SRPS } & \multirow{2}{*}{$\begin{array}{l}\mathrm{p} \text { for } \\
\text { trend }\end{array}$} & \multirow[b]{2}{*}{$\mathrm{p}$ for int } \\
\hline & $\begin{array}{c}\text { Low }^{2} \\
(\mathrm{n}=1,558)\end{array}$ & $\begin{array}{l}\text { Moderate }^{2} \\
(\mathrm{n}=3,247)\end{array}$ & $\operatorname{High}^{2}(\mathrm{n}=782)$ & & & $\operatorname{Low}^{2}(\mathrm{n}=641)$ & $\begin{array}{l}\text { Moderate }^{2} \\
(\mathrm{n}=1,691)\end{array}$ & $\operatorname{High}^{2}(\mathrm{n}=386)$ & & \\
\hline Rice & & & & & 0.37 & & & & & 0.11 \\
\hline Non-exercisers $^{3}$ & $\begin{array}{c}218.5 \\
(213.4-223.6)\end{array}$ & $\begin{array}{c}217.1 \\
(213.8-220.4)\end{array}$ & $\begin{array}{c}213.9 \\
(207.6-220.3)\end{array}$ & 0.28 & & $\begin{array}{c}210.0 \\
(202.8-217.3)\end{array}$ & $\begin{array}{c}208.5 \\
(204.3-212.8)\end{array}$ & $\begin{array}{c}211.9 \\
(203.7-220.0)\end{array}$ & 0.77 & \\
\hline Exercisers $^{4}$ & $\begin{array}{c}209.4 \\
(204.8-214.0)\end{array}$ & $\begin{array}{c}210.0 \\
(206.6-213.3)\end{array}$ & $\begin{array}{c}210.1 \\
(202.5-217.6)\end{array}$ & 0.79 & & $\begin{array}{c}199.7 \\
(192.2-207.2)\end{array}$ & $\begin{array}{c}200.0 \\
(195.2-204.9)\end{array}$ & $\begin{array}{c}210.4 \\
(198.8-222.0)\end{array}$ & 0.19 & \\
\hline Bread & & & & & 0.92 & & & & & 0.36 \\
\hline Non-exercisers & $\begin{array}{c}22.0 \\
(20.8-23.3)\end{array}$ & $\begin{array}{c}20.8 \\
(20.0-21.6)\end{array}$ & $\begin{array}{c}21.5 \\
(19.9-23.1)\end{array}$ & 0.43 & & $\begin{array}{c}28.7 \\
(26.5-30.8)\end{array}$ & $\begin{array}{c}29.3 \\
(28.1-30.6)\end{array}$ & $\begin{array}{c}27.2 \\
(24.8-29.7)\end{array}$ & 0.56 & \\
\hline Exercisers & $\begin{array}{c}21.1 \\
(19.9-22.3)\end{array}$ & $\begin{array}{c}20.4 \\
(19.5-21.3)\end{array}$ & $\begin{array}{c}20.8(18.8- \\
22.7)\end{array}$ & 0.58 & & $\begin{array}{c}28.8 \\
(26.6-31.1)\end{array}$ & $\begin{array}{c}29.7 \\
(28.2-31.1)\end{array}$ & $\begin{array}{c}27.7 \\
(24.2-31.2)\end{array}$ & 0.96 & \\
\hline Buckwheat noodles & & & & $\mathrm{v}$ & 0.10 & & & & & 0.15 \\
\hline Non-exercisers & $\begin{array}{c}14.8 \\
(13.6-16.0)\end{array}$ & $\begin{array}{c}16.1 \\
(15.3-16.9)\end{array}$ & $\begin{array}{c}15.8 \\
(14.3-17.3)\end{array}$ & 0.21 & & $\begin{array}{c}9.6 \\
(8.3-10.9)\end{array}$ & $\begin{array}{c}9.6 \\
(8.8-10.3)\end{array}$ & $\begin{array}{c}9.3 \\
(7.9-10.8)\end{array}$ & 0.96 & \\
\hline Exercisers & $\begin{array}{c}16.9 \\
(15.8-18.0)\end{array}$ & $\begin{array}{c}16.3 \\
(15.5-17.1)\end{array}$ & $\begin{array}{c}15.4 \\
(13.7-17.2)\end{array}$ & 0.20 & & $\begin{array}{c}10.0 \\
(8.7-11.3)\end{array}$ & $\begin{array}{c}10.5 \\
(9.7-11.3)\end{array}$ & $\begin{array}{c}10.9 \\
(8.9-13.0)\end{array}$ & 0.48 & \\
\hline \multicolumn{2}{|c|}{ Japanese wheat noodles } & & & & 0.85 & & & & & 0.80 \\
\hline Non-exercisers & $\begin{array}{c}12.0 \\
(11.0-13.0)\end{array}$ & $\begin{array}{c}13.3 \\
(12.6-13.9)\end{array}$ & $\begin{array}{c}12.2 \\
(11.0-13.5)\end{array}$ & 0.52 & & $\begin{array}{c}12.0 \\
(10.6-13.5)\end{array}$ & $\begin{array}{c}13.0 \\
(12.1-13.8)\end{array}$ & $\begin{array}{c}14.0 \\
(12.4-15.6)\end{array}$ & 0.13 & \\
\hline Exercisers & $\begin{array}{c}12.7 \\
(11.8-13.6)\end{array}$ & $\begin{array}{c}12.7 \\
(12.1-13.4)\end{array}$ & $\begin{array}{c}13.1 \\
(11.6-14.6)\end{array}$ & 0.70 & & $\begin{array}{c}12.7 \\
(11.2-14.1)\end{array}$ & $\begin{array}{c}13.0 \\
(12.1-13.9)\end{array}$ & $\begin{array}{c}13.1 \\
(10.9-15.3)\end{array}$ & 0.69 & \\
\hline Chinese, noodles & & & & & 0.83 & & & & & 0.24 \\
\hline Non-exercisers & $\begin{array}{c}17.0 \\
(15.8-18.1)\end{array}$ & $\begin{array}{c}17.6 \\
(16.9-18.4)\end{array}$ & $\begin{array}{c}17.5 \\
(16.1-19.0)\end{array}$ & 0.53 & & $\begin{array}{c}10.2 \\
(8.9-11.5)\end{array}$ & $\begin{array}{c}10.4 \\
(9.7-11.2)\end{array}$ & $\begin{array}{c}10.9 \\
(9.5-12.4)\end{array}$ & 0.42 & \\
\hline Exercisers & $\begin{array}{c}14.0 \\
(13.1-14.9)\end{array}$ & $\begin{array}{c}14.2 \\
(13.6-14.9)\end{array}$ & $\begin{array}{c}14.4 \\
(12.9-15.8)\end{array}$ & 0.65 & & $\begin{array}{c}8.4 \\
(7.3-9.5)\end{array}$ & $\begin{array}{c}9.1 \\
(8.4-9.8)\end{array}$ & $\begin{array}{c}9.4 \\
(7.8-11.1)\end{array}$ & 0.25 & \\
\hline \multicolumn{2}{|c|}{ Spaghetti and macaroni } & & & & 0.11 & & & & & 0.63 \\
\hline Non-exercisers & $\begin{array}{c}10.3 \\
(9.6-11.0)\end{array}$ & $\begin{array}{c}10.6 \\
(10.2-11.1)\end{array}$ & $\begin{array}{c}11.3 \\
(10.4-12.2)\end{array}$ & 0.10 & & $\begin{array}{c}11.5 \\
(10.4-12.6)\end{array}$ & $\begin{array}{c}11.0 \\
(10.3-11.6)\end{array}$ & $\begin{array}{c}11.7 \\
(10.5-12.9)\end{array}$ & 0.96 & \\
\hline Exercisers & $9.9(9.3-10.6)$ & $\begin{array}{c}10.1 \\
(9.6-10.6)\end{array}$ & $\begin{array}{c}9.2 \\
(8.2-10.3)\end{array}$ & 0.49 & & $\begin{array}{c}10.4 \\
(9.2-11.6)\end{array}$ & $\begin{array}{c}10.3 \\
(9.5-11.1)\end{array}$ & $\begin{array}{c}10.3 \\
(8.4-12.1)\end{array}$ & 0.95 & \\
\hline Chicken & & & & & 0.76 & & & & & 0.46 \\
\hline Non-exercisers & $\begin{array}{c}308.3 \\
(292.9-323.7)\end{array}$ & $\begin{array}{c}307.6 \\
(297.6-317.7)\end{array}$ & $\begin{array}{c}306.9 \\
(287.7-326.1)\end{array}$ & 0.88 & & $\begin{array}{c}287.9 \\
(265.5-310.2)\end{array}$ & $\begin{array}{c}300.1 \\
(286.9-313.2)\end{array}$ & $\begin{array}{c}292.5 \\
(267.4-317.5)\end{array}$ & 0.71 & \\
\hline Exercisers & $\begin{array}{c}295.7 \\
(282.8-308.7)\end{array}$ & $\begin{array}{c}300.0 \\
(290.5-309.5)\end{array}$ & $\begin{array}{c}299.4 \\
(278.3-320.6)\end{array}$ & 0.67 & & $\begin{array}{c}286.8 \\
(265.1-308.6)\end{array}$ & $\begin{array}{c}287.8 \\
(273.9-301.8)\end{array}$ & $\begin{array}{c}265.8 \\
(232.1-299.4)\end{array}$ & 0.48 & \\
\hline Pork /beef & & & & & 0.79 & & & & & $0.03^{*}$ \\
\hline Non-exercisers & $\begin{array}{c}14.0 \\
(13.3-14.6)\end{array}$ & $\begin{array}{c}14.4 \\
(14.0-14.8)\end{array}$ & $\begin{array}{c}14.8 \\
(14.0-15.6)\end{array}$ & 0.12 & & $\begin{array}{c}17.7 \\
(16.6-18.7)\end{array}$ & $\begin{array}{c}17.3 \\
(16.7-17.9)\end{array}$ & $\begin{array}{c}17.6 \\
(16.4-18.8)\end{array}$ & 0.84 & \\
\hline Exercisers & $\begin{array}{c}13.7 \\
(13.1-14.2)\end{array}$ & $\begin{array}{c}14.0 \\
(13.6-14.4)\end{array}$ & $\begin{array}{c}14.1 \\
(13.2-15.0)\end{array}$ & 0.33 & & $\begin{array}{c}17.8 \\
(16.7-19.0)\end{array}$ & $\begin{array}{c}16.8 \\
(16.1-17.6)\end{array}$ & $\begin{array}{c}15.5 \\
(13.7-17.3)\end{array}$ & $0.02 *$ & \\
\hline \multicolumn{3}{|c|}{ Ham, sausages, and bacon } & & & 0.24 & & & & & 0.39 \\
\hline Non-exercisers & $\begin{array}{c}3.4 \\
(3.2-3.7)\end{array}$ & $\begin{array}{c}3.8 \\
(3.6-3.9)\end{array}$ & $\begin{array}{c}3.7 \\
(3.4-4.0)\end{array}$ & 0.12 & & $3.8(3.4-4.2)$ & $4.2(4.0-4.5)$ & $3.8(3.3-4.2)$ & 0.97 & \\
\hline Exercisers & $\begin{array}{c}3.7 \\
(3.4-3.9)\end{array}$ & $\begin{array}{c}3.7 \\
(3.5-3.9)\end{array}$ & $\begin{array}{c}3.6 \\
(3.2-4.0)\end{array}$ & 0.89 & & $4.5(4.0-4.9)$ & $4.0(3.8-4.3)$ & $\begin{array}{c}3.6 \\
(2.9-4.2)\end{array}$ & $0.01^{*}$ & \\
\hline Liver & & & & & 0.76 & & & & & 0.55 \\
\hline Non-exercisers & $\begin{array}{c}1.1 \\
(0.9-1.2)\end{array}$ & $\begin{array}{c}1.1 \\
(1.0-1.2)\end{array}$ & $\begin{array}{c}0.9 \\
(0.7-1.0)\end{array}$ & 0.09 & & $\begin{array}{c}0.8 \\
(0.7-1.0)\end{array}$ & $\begin{array}{c}0.9 \\
(0.8-1.0)\end{array}$ & $1.0(0.8-1.2)$ & 0.34 & \\
\hline Exercisers & $\begin{array}{c}1.2 \\
(1.0-1.3)\end{array}$ & $\begin{array}{c}1.2 \\
(1.1-1.2)\end{array}$ & $\begin{array}{c}1.0 \\
(0.8-1.2)\end{array}$ & 0.29 & & $\begin{array}{c}0.9 \\
(0.7-1.0)\end{array}$ & $0.9(0.8-1.0)$ & $1.0(0.7-1.2)$ & 0.54 & \\
\hline \multicolumn{2}{|c|}{ Squid, octopus, shrimp, and clams } & & & & 0.33 & & & & & 0.51 \\
\hline Non-exercisers & $6.7(6.3-7.1)$ & $7.3(7.0-7.6)$ & $\begin{array}{c}6.6 \\
(6.1-7.2)\end{array}$ & 0.93 & & $6.2(5.6-6.8)$ & $6.8(6.4-7.1)$ & $6.7(6.0-7.4)$ & 0.34 & \\
\hline Exercisers & $6.7(6.4-7.1)$ & $7.1(6.9-7.4)$ & $7.1(6.5-7.8)$ & 0.17 & & $6.6(6.0-7.2)$ & $6.8(6.4-7.2)$ & $7.0(6.0-7.9)$ & 0.47 & \\
\hline \multicolumn{2}{|c|}{ Small fish with bones } & & & & 0.33 & & & & & 0.45 \\
\hline Non-exercisers & $2.1(1.9-2.3)$ & $2.1(2.0-2.3)$ & $2.1(1.8-2.3)$ & 0.84 & & $2.1(1.8-2.5)$ & $2.2(2.0-2.4)$ & $2.6(2.2-3.0)$ & 0.15 & \\
\hline Exercisers & $2.7(2.4-2.9)$ & $2.6(2.4-2.7)$ & $2.4(2.0-2.8)$ & 0.25 & & $3.1(2.7-3.6)$ & $2.6(2.4-2.9)$ & $2.6(2.0-3.3)$ & 0.07 & \\
\hline Canned tuna & & & & & 0.82 & & & & & 0.32 \\
\hline Non-exercisers & $1.5(1.3-1.6)$ & $1.5(1.4-1.6)$ & $1.5(1.3-1.7)$ & 0.78 & & $1.6(1.4-1.8)$ & $1.6(1.5-1.8)$ & $1.5(1.3-1.7)$ & 0.85 & \\
\hline Exercisers & $1.4(1.3-1.5)$ & $1.4(1.3-1.5)$ & $1.4(1.1-1.6)$ & 0.91 & & $1.8(1.6-2.0)$ & $1.6(1.4-1.7)$ & $1.4(1.0-1.7)$ & $0.04 *$ & \\
\hline \multicolumn{2}{|c|}{ Dried fish and salted fish } & & & & 0.34 & & & & & 0.09 \\
\hline Non-exercisers & $5.4(5.0-5.8)$ & $5.7(5.5-6.0)$ & $5.7(5.2-6.2)$ & 0.34 & & $6.4(5.7-7.0)$ & $6.3(6.0-6.7)$ & $6.3(5.6-7.1)$ & 0.90 & \\
\hline Exercisers & $6.0(5.7-6.4)$ & $6.1(5.8-6.3)$ & $5.8(5.2-6.4)$ & 0.62 & & $6.7(5.9-7.4)$ & $7.3(6.9-7.8)$ & $6.5(5.3-7.6)$ & 0.72 & \\
\hline Oily fish & & & & & 0.71 & & & & & 0.98 \\
\hline Non-exercisers & $7.2(6.8-7.6)$ & $7.2(6.9-7.5)$ & $7.6(7.0-8.1)$ & 0.33 & & $7.9(7.2-8.6)$ & $7.8(7.4-8.2)$ & $7.6(6.8-8.4)$ & 0.52 & \\
\hline
\end{tabular}


Table 2 (continued). Interactions between SRPS and Habitual Exercise Level in the Dietary Intake of Food in Middle-Aged Japanese Men And Women ${ }^{1}$

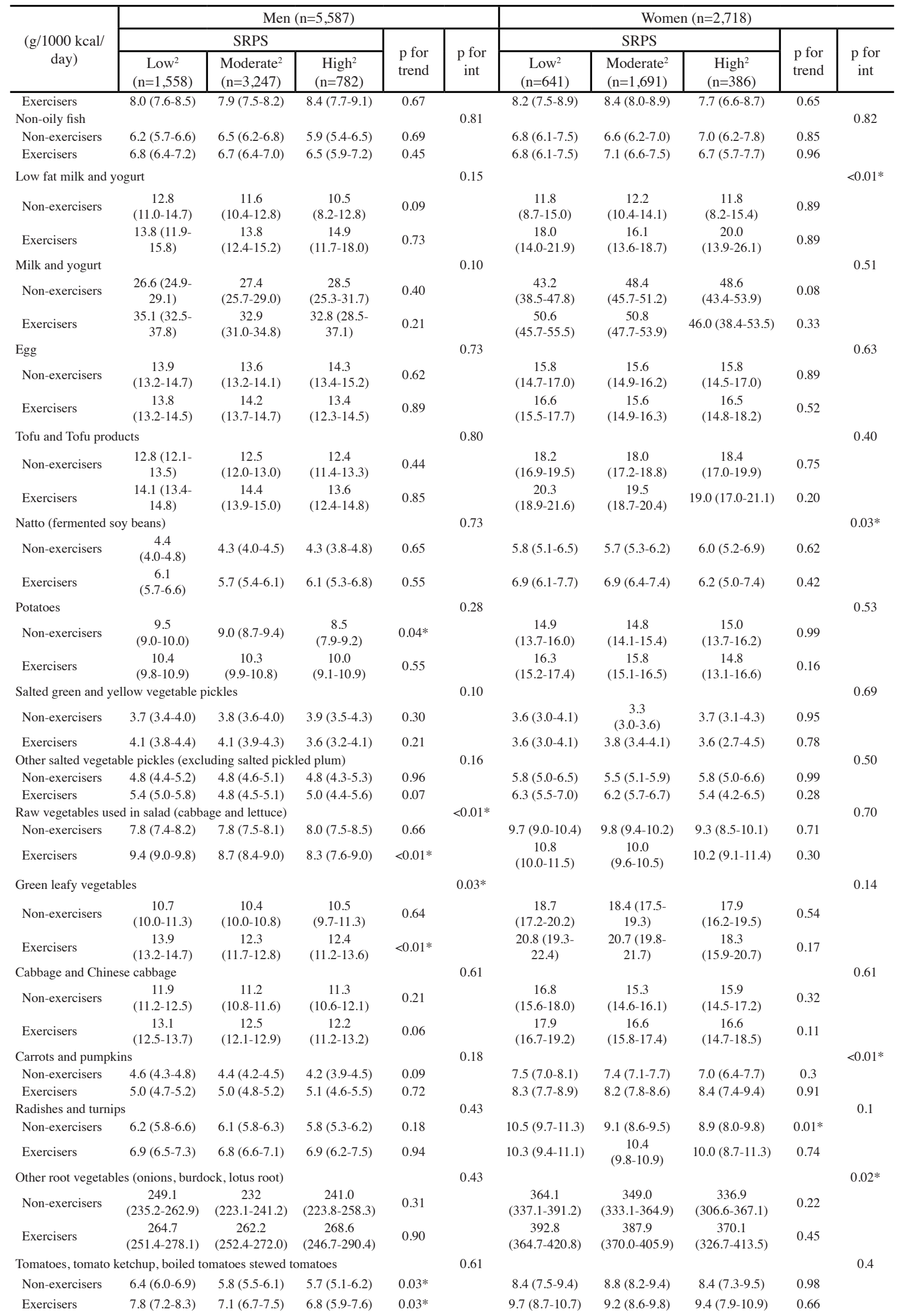


DOI:http://dx.doi.org/10.7314/APJCP.2016.17.4.2007

Impact of Interaction of Psychological Stress with Habitual Exercise on Dietary Intake

Table 2 (continued). Interactions between SRPS and Habitual Exercise Level in the Dietary Intake of Food in Middle-Aged Japanese Men And Women ${ }^{1}$

\begin{tabular}{|c|c|c|c|c|c|c|c|c|c|c|}
\hline \multirow{3}{*}{$\begin{array}{c}\text { (g/1000 kcal/ } \\
\text { day) }\end{array}$} & \multicolumn{5}{|c|}{$\operatorname{Men}(n=5,587)$} & \multicolumn{5}{|c|}{ Women $(n=2,718)$} \\
\hline & \multicolumn{3}{|c|}{ SRPS } & \multirow{2}{*}{$\begin{array}{l}\mathrm{p} \text { for } \\
\text { trend }\end{array}$} & \multirow{2}{*}{$\begin{array}{l}\mathrm{p} \text { for } \\
\text { int }\end{array}$} & \multicolumn{3}{|c|}{ SRPS } & \multirow{2}{*}{$\begin{array}{l}\mathrm{p} \text { for } \\
\text { trend }\end{array}$} & \multirow{2}{*}{$\begin{array}{l}\mathrm{p} \text { for } \\
\text { int }\end{array}$} \\
\hline & $\begin{array}{c}\text { Low }^{2} \\
(n=1,558)\end{array}$ & $\begin{array}{l}\text { Moderate }^{2} \\
(\mathrm{n}=3,247)\end{array}$ & $\begin{array}{l}\text { High }^{2} \\
(n=782)\end{array}$ & & & $\begin{array}{c}\text { Low }^{2} \\
(n=641)\end{array}$ & $\begin{array}{l}\text { Moderate }^{2} \\
(\mathrm{n}=1,691)\end{array}$ & $\begin{array}{c}\text { High }^{2} \\
(n=386)\end{array}$ & & \\
\hline \multicolumn{5}{|l|}{ Mushrooms } & \multicolumn{5}{|l|}{0.06} & $0.02 *$ \\
\hline Non-exercisers & $5.0(4.7-5.3)$ & $\begin{array}{c}4.8 \\
(4.6-5.0)\end{array}$ & $4.7(4.3-5.1)$ & 0.32 & & $8.0(7.4-8.7)$ & $8.3(7.9-8.7)$ & $8.9(8.1-9.6)$ & 0.1 & \\
\hline Exercisers & $5.4(5.0-5.7)$ & $\begin{array}{c}5.5 \\
(5.3-5.7)\end{array}$ & $5.8(5.3-6.3)$ & 0.17 & & $9.6(8.9-10.3)$ & $9.6(9.1-10.0)$ & $8.8(7.8-9.9)$ & 0.35 & \\
\hline \multicolumn{5}{|l|}{ Seaweeds } & 0.41 & & & & & $0.04 *$ \\
\hline Non-exercisers & $5.9(5.5-6.3)$ & $\begin{array}{c}5.5 \\
(5.2-5.7)\end{array}$ & $5.2(4.7-5.7)$ & $0.04 *$ & & $8.2(7.5-9.0)$ & $8.4(7.9-8.8)$ & $8.0(7.1-8.9)$ & 0.84 & \\
\hline Exercisers & $6.7(6.3-7.1)$ & $6.5(6.2-6.8)$ & $6.5(5.8-7.1)$ & 0.43 & & $9.6(8.8-10.4)$ & $9.6(9.1-10.1)$ & $8.9(7.6-10.1)$ & 0.57 & \\
\hline \multicolumn{4}{|c|}{ Citrus fruit, including oranges } & & 0.98 & & & & & 0.54 \\
\hline Non-exercisers & $\begin{array}{c}10.0 \\
(9.0-11.1)\end{array}$ & $\begin{array}{c}9.9 \\
(9.2-10.6)\end{array}$ & $9.5(8.2-10.8)$ & 0.51 & & $\begin{array}{c}18.3 \\
(15.9-20.7)\end{array}$ & $\begin{array}{c}16.2 \\
(14.8-17.6)\end{array}$ & $\begin{array}{c}17.1 \\
(14.5-19.8)\end{array}$ & 0.38 & \\
\hline Exercisers & $\begin{array}{c}11.6 \\
(10.6-12.6)\end{array}$ & $\begin{array}{c}11.4 \\
(10.7-12.1)\end{array}$ & $\begin{array}{c}10.8 \\
(9.2-12.5)\end{array}$ & 0.50 & & $\begin{array}{c}22.0 \\
(19.3-24.7)\end{array}$ & $\begin{array}{c}20.7 \\
(19.0-22.5)\end{array}$ & $\begin{array}{c}17.3 \\
(13.1-21.5)\end{array}$ & 0.08 & \\
\hline \multicolumn{5}{|c|}{ Strawberries, persimmons, and kiwi fruits } & 0.40 & & & & & 0.99 \\
\hline Non-exercisers & $5.4(4.8-6.0)$ & $4.9(4.5-5.3)$ & $5.4(4.6-6.1)$ & 0.84 & & $10.0(8.4-11.6)$ & $9.3(8.4-10.2)$ & $9.1(7.3-10.9)$ & 0.4 & \\
\hline Exercisers & $6.4(5.7-7.0)$ & $6.1(5.6-6.6)$ & $5.6(4.6-6.7)$ & 0.24 & & $11.6(9.9-13.2)$ & $\begin{array}{c}10.8 \\
(9.7-11.9)\end{array}$ & $9.0(6.5-11.6)$ & 0.14 & \\
\hline \multicolumn{5}{|l|}{ Other fruits } & 0.71 & & & & & 0.47 \\
\hline Non-exercisers & $\begin{array}{c}13.5 \\
(12.5-14.6)\end{array}$ & $\begin{array}{c}12.1 \\
(11.5-12.8)\end{array}$ & $\begin{array}{c}12.9 \\
(11.6-14.2)\end{array}$ & 0.30 & & $\begin{array}{c}23.8 \\
(21.5-26.1)\end{array}$ & $\begin{array}{c}22.0 \\
(20.6-23.4)\end{array}$ & $\begin{array}{c}21.5 \\
(18.9-24.1)\end{array}$ & 0.16 & \\
\hline Exercisers & $\begin{array}{c}16.8 \\
(15.7-17.9)\end{array}$ & $\begin{array}{c}15.4 \\
(14.6-16.3)\end{array}$ & $\begin{array}{c}16.0 \\
(14.1-17.8)\end{array}$ & 0.17 & & $\begin{array}{c}25.1 \\
(22.7-27.5)\end{array}$ & $\begin{array}{c}25.0 \\
(23.4-26.5)\end{array}$ & $\begin{array}{c}24.3 \\
(20.6-28.0)\end{array}$ & 0.68 & \\
\hline \multicolumn{5}{|c|}{ Cakes, cookies, and biscuits } & 0.86 & & & & & 0.28 \\
\hline Non-exercisers & $3.9(3.5-4.3)$ & $4.5(4.2-4.7)$ & $5.1(4.6-5.5)$ & $<0.001^{*}$ & & $8.6(7.8-9.5)$ & $8.6(8.1-9.0)$ & $8.1(7.2-9.1)$ & 0.44 & \\
\hline Exercisers & $3.9(3.6-4.3)$ & $4.5(4.2-4.7)$ & $5.1(4.5-5.7)$ & $<0.001^{*}$ & & $7.6(6.7-8.5)$ & $8.3(7.7-8.8)$ & $8.7(7.4-10.0)$ & 0.14 & \\
\hline \multicolumn{4}{|c|}{ Japanese-style sweets } & & 0.88 & & & & & 0.78 \\
\hline Non-exercisers & $1.4(1.3-1.6)$ & $1.5(1.4-1.6)$ & $1.5(1.3-1.7)$ & 0.36 & & $2.7(2.4-3.0)$ & $2.7(2.5-2.9)$ & $2.5(2.1-2.8)$ & 0.32 & \\
\hline Exercisers & $1.5(1.4-1.7)$ & $1.6(1.5-1.7)$ & $1.7(1.4-1.9)$ & 0.42 & & $3.4(3.0-3.8)$ & $2.8(2.6-3.1)$ & $3.3(2.6-3.9)$ & 0.28 & \\
\hline Rice crackers, rice & cakes, and Japan & ese-style pancake & & & 0.51 & & & & & 0.93 \\
\hline Non-exercisers & $2.4(2.2-2.6)$ & $2.6(2.4-2.7)$ & $2.7(2.5-3.0)$ & 0.07 & & $3.8(3.4-4.3)$ & $3.9(3.7-4.2)$ & $3.8(3.3-4.3)$ & 0.87 & \\
\hline Exercisers & $2.3(2.1-2.5)$ & $2.5(2.4-2.6)$ & $2.8(2.5-3.1)$ & $0.02 *$ & & $3.7(3.2-4.1)$ & $3.8(3.6-4.1)$ & $4.0(3.4-4.6)$ & 0.39 & \\
\hline Ice cream & & & & & 0.77 & & & & & 0.23 \\
\hline Non-exercisers & $8.0(7.1-8.9)$ & $8.2(7.6-8.7)$ & $8.1(7.0-9.2)$ & 0.90 & & $10.6(9.0-12.2)$ & $9.5(8.5-10.4)$ & $9.6(7.8-11.4)$ & 0.32 & \\
\hline Exercisers & $8.3(7.4-9.1)$ & $8.4(7.8-9.0)$ & $8.0(6.7-9.4)$ & 0.94 & & $7.0(5.6-8.4)$ & $7.7(6.8-8.6)$ & $10.2(8.0-12.4)$ & $0.04 *$ & \\
\hline Mayonnaise and s & ad dressing & & & & 0.31 & & & & & 0.51 \\
\hline Non-exercisers & $2.8(2.7-3.0)$ & $2.7(2.6-2.8)$ & $2.7(2.5-2.9)$ & 0.29 & & $3.3(3.0-3.5)$ & $3.3(3.2-3.5)$ & $3.2(2.9-3.5)$ & 0.73 & \\
\hline Exercisers & $2.9(2.8-3.1)$ & $2.7(2.6-2.9)$ & $2.7(2.4-2.9)$ & $0.03 *$ & & $3.2(3.0-3.5)$ & $3.3(3.1-3.4)$ & $3.1(2.7-3.6)$ & 0.86 & \\
\hline Green tea & & & & & 0.55 & & & & & 0.20 \\
\hline Non-exercisers & $\begin{array}{c}173.5 \\
(165.5-181.4)\end{array}$ & $\begin{array}{c}177.3 \\
(172.1-182.4)\end{array}$ & $\begin{array}{c}174.7 \\
(165-184.6)\end{array}$ & 0.77 & & $\begin{array}{c}206.3 \\
(193.6-219.0)\end{array}$ & $\begin{array}{c}219.7 \\
(212.2-227.1)\end{array}$ & $\begin{array}{c}233.7 \\
(219.5-248.0)\end{array}$ & $<0.01 *$ & \\
\hline Exercisers & $\begin{array}{c}183.3 \\
(176.1-190.5)\end{array}$ & $\begin{array}{c}181.0 \\
(175.7-186.2)\end{array}$ & $\begin{array}{c}194.9 \\
(183-206.6)\end{array}$ & 0.26 & & $\begin{array}{c}218.1 \\
(205.6-230.6)\end{array}$ & $\begin{array}{c}219.8 \\
(211.9-227.8)\end{array}$ & $\begin{array}{c}213.8 \\
(194.5-233.1)\end{array}$ & 0.86 & \\
\hline Black and oolong & a (including oth & r Chinese tea) & & & 0.10 & & & & & 0.99 \\
\hline Non-exercisers & $\begin{array}{c}38.4 \\
(33.2-43.7)\end{array}$ & $\begin{array}{c}38.8 \\
(35.4-42.2)\end{array}$ & $\begin{array}{c}40 \\
(33.5-46.5)\end{array}$ & 0.71 & & $\begin{array}{c}58.6 \\
(49.1-68.1)\end{array}$ & $\begin{array}{c}54.2 \\
(48.6-59.8)\end{array}$ & $\begin{array}{c}47.8 \\
(37.1-58.5)\end{array}$ & 0.19 & \\
\hline Exercisers & $\begin{array}{c}39.7 \\
(35.2-44.2)\end{array}$ & $\begin{array}{c}36.2 \\
(32.9-39.5)\end{array}$ & $\begin{array}{c}32 \\
(24.6-39.3)\end{array}$ & 0.07 & & $\begin{array}{c}50.3 \\
(41.5-59.2)\end{array}$ & $\begin{array}{c}49.8 \\
(44.1-55.4)\end{array}$ & $\begin{array}{c}55.7 \\
(42.0-69.4)\end{array}$ & 0.67 & \\
\hline Coffee & & & & & 0.60 & & & & & 0.13 \\
\hline Non-exercisers & $\begin{array}{c}117.0 \\
(109.6-124.4)\end{array}$ & $\begin{array}{c}115.2 \\
(110.3-120.0)\end{array}$ & $\begin{array}{c}113.8 \\
(104.6-123.1)\end{array}$ & 0.56 & & $\begin{array}{c}92.6 \\
(82.3-102.8)\end{array}$ & $\begin{array}{c}93.7 \\
(87.7-99.8)\end{array}$ & $\begin{array}{c}86.5 \\
(75.0-98.1)\end{array}$ & 0.41 & \\
\hline Exercisers & $\begin{array}{c}103.2 \\
(96.9-109.6)\end{array}$ & $\begin{array}{c}105.7 \\
(101.0-110.4)\end{array}$ & $\begin{array}{c}90.1 \\
(80.3-101.1)\end{array}$ & 0.20 & & $\begin{array}{c}98.2 \\
(88.1-108.2)\end{array}$ & $\begin{array}{c}98.0 \\
(91.6-104.5)\end{array}$ & $\begin{array}{c}93.6 \\
(78-109.2)\end{array}$ & 0.77 & \\
\hline Soda & & & & & 0.55 & & & & & 0.09 \\
\hline Non-exercisers & $\begin{array}{c}19.2 \\
(16.7-21.8)\end{array}$ & $\begin{array}{c}19.5(17.9- \\
21.2)\end{array}$ & $\begin{array}{c}22 \\
(18.8-25.1)\end{array}$ & 0.22 & & $9.7(7.4-11.9)$ & $\begin{array}{c}10.5 \\
(9.1-11.8)\end{array}$ & $\begin{array}{c}7.5 \\
(5.0-10.0)\end{array}$ & 0.27 & \\
\hline Exercisers & $\begin{array}{c}17.4 \\
(15.1-19.7)\end{array}$ & $\begin{array}{c}19.3(17.6- \\
21.0)\end{array}$ & $\begin{array}{c}21.9 \\
(17.8-25.4)\end{array}$ & 0.05 & & $10.9(8.1-13.7)$ & $\begin{array}{c}11.1 \\
(9.3-12.9)\end{array}$ & $\begin{array}{c}10.9 \\
(6.5-15.2)\end{array}$ & 0.85 & \\
\hline Fruit juice and ve & table juice & & & & $0.02 *$ & & & & & 0.45 \\
\hline Non-exercisers & $\begin{array}{c}15.8 \\
(13.7-18.0)\end{array}$ & $\begin{array}{c}15.2(13.8- \\
16.6)\end{array}$ & $\begin{array}{c}13.0 \\
(10.4-15.7)\end{array}$ & 0.12 & & $\begin{array}{c}12.1 \\
(9.2-14.9)\end{array}$ & $\begin{array}{c}14.9 \\
(13.2-16.5)\end{array}$ & $\begin{array}{c}15.4 \\
(12.3-18.6)\end{array}$ & 0.10 & \\
\hline Exercisers & $\begin{array}{c}16.0 \\
(13.7-18.3)\end{array}$ & $\begin{array}{c}19.8 \\
(18.1-21.5)\end{array}$ & $\begin{array}{c}17 \\
(13.5-21.1)\end{array}$ & 0.15 & & $\begin{array}{c}17.3 \\
(13.6-21.1)\end{array}$ & $\begin{array}{c}15.7 \\
(13.3-18.1)\end{array}$ & $\begin{array}{c}16.2 \\
(10.3-22.0)\end{array}$ & 0.53 & \\
\hline Sake & & & & & 0.64 & & & & & 0.57 \\
\hline Non-exercisers & $\begin{array}{c}13.6 \\
(10.8-16.4)\end{array}$ & $\begin{array}{c}12.7 \\
(10.9-14.5)\end{array}$ & $\begin{array}{c}15.2 \\
(11.7-18.7)\end{array}$ & 0.56 & & $\begin{array}{c}1.3 \\
(-0.3-2.9)\end{array}$ & $2.0(1.1-3.0)$ & $\begin{array}{c}3.7 \\
(2.0-5.5)\end{array}$ & 0.05 & \\
\hline Exercisers & $\begin{array}{c}13.1 \\
(10.8-15.5)\end{array}$ & $\begin{array}{c}12.6 \\
(10.9-14.3)\end{array}$ & $\begin{array}{c}13.2 \\
(9.4-17.0)\end{array}$ & 0.91 & & $\begin{array}{c}2.6 \\
(1.0-4.1)\end{array}$ & $1.9(0.9-2.9)$ & $\begin{array}{c}3.4 \\
(1.1-5.8)\end{array}$ & 0.84 & \\
\hline Beer & & & & & 0.15 & & & & & 0.53 \\
\hline Non-exercisers & $\begin{array}{c}122.0 \\
(112.3-131.7)\end{array}$ & $\begin{array}{c}109 \\
(102.2-114.9)\end{array}$ & $\begin{array}{c}107 \\
(95.1-119.2)\end{array}$ & $0.04 *$ & & $\begin{array}{c}40.1 \\
(29.6-50.6)\end{array}$ & $\begin{array}{c}38.0 \\
(31.8-44.2)\end{array}$ & $\begin{array}{c}57.7 \\
(45.9-69.5)\end{array}$ & 0.06 & \\
\hline Exercisers & $\begin{array}{c}110.7 \\
(102.9-118.5) \\
\end{array}$ & $\begin{array}{c}108.0 \\
(102.3-113.7) \\
\end{array}$ & $\begin{array}{c}113 \\
(101-126.2) \\
\end{array}$ & 0.91 & & $\begin{array}{c}40.5 \\
(30.9-50.1) \\
\end{array}$ & $\begin{array}{c}44.6 \\
(38.5-50.8) \\
\end{array}$ & $\begin{array}{c}40.9 \\
(26.0-55.8) \\
\end{array}$ & 0.78 & \\
\hline
\end{tabular}


Kaori Endoh et al

Table 2 (continued). Interactions between SRPS and Habitual Exercise Level in the Dietary Intake of Food in Middle-Aged Japanese Men And Women ${ }^{1}$

\begin{tabular}{|c|c|c|c|c|c|c|c|c|c|c|}
\hline \multirow{3}{*}{$\begin{array}{c}\text { (g/1000 kcal/ } \\
\text { day) }\end{array}$} & \multicolumn{5}{|c|}{ Men $(n=5,587)$} & \multicolumn{5}{|c|}{ Women $(n=2,718)$} \\
\hline & \multicolumn{3}{|c|}{ SRPS } & \multirow[b]{2}{*}{$\begin{array}{l}\mathrm{p} \text { for } \\
\text { trend }\end{array}$} & \multirow[b]{2}{*}{$\begin{array}{l}\mathrm{p} \text { for } \\
\text { int }\end{array}$} & \multicolumn{3}{|c|}{ SRPS } & \multirow[b]{2}{*}{$\begin{array}{l}\mathrm{p} \text { for } \\
\text { trend }\end{array}$} & \multirow[b]{2}{*}{$\begin{array}{l}\mathrm{p} \text { for } \\
\text { int }\end{array}$} \\
\hline & $\begin{array}{c}\text { Low }^{2} \\
(n=1,558) \\
\end{array}$ & $\begin{array}{l}\text { Moderate }^{2} \\
(n=3,247)\end{array}$ & $\begin{array}{c}\text { High }^{2} \\
(\mathrm{n}=782) \\
\end{array}$ & & & $\begin{array}{c}\text { Low }^{2} \\
(n=641)\end{array}$ & $\begin{array}{l}\text { Moderate }^{2} \\
(\mathrm{n}=1,691)\end{array}$ & $\begin{array}{c}\text { High }^{2} \\
(\mathrm{n}=386)\end{array}$ & & \\
\hline \multicolumn{5}{|c|}{ Shochu and shochu mixed with water or a carbonated beverage } & 0.66 & & & & & 0.52 \\
\hline Non-exercisers & $\begin{array}{c}33.4 \\
(30.1-36.7)\end{array}$ & $\begin{array}{c}32.4 \\
(30.2-34.5)\end{array}$ & $\begin{array}{c}32.0 \\
(27.9-36.1)\end{array}$ & 0.59 & & $\begin{array}{c}8.2 \\
(5.6-10.9)\end{array}$ & $\begin{array}{c}7.4 \\
(5.9-9.0)\end{array}$ & $\begin{array}{c}6.0 \\
(3.1-9.0)\end{array}$ & 0.31 & \\
\hline Exercisers & $\begin{array}{c}32.7 \\
(29.8-35.7)\end{array}$ & $\begin{array}{c}31.2 \\
(29.1-33.4)\end{array}$ & $\begin{array}{c}33.4 \\
(28.6-38.2)\end{array}$ & 0.72 & & $\begin{array}{c}6.6 \\
(4.1-9.0)\end{array}$ & $\begin{array}{c}7.0 \\
(5.4-8.6)\end{array}$ & $\begin{array}{c}7.8 \\
(3.9-11.6)\end{array}$ & 0.59 & \\
\hline Whiskey & & & & & 0.10 & & & & & 0.54 \\
\hline Non-exercisers & $\begin{array}{c}2.7 \\
(1.9-3.6)\end{array}$ & $\begin{array}{c}2.1 \\
(1.5-2.6)\end{array}$ & $\begin{array}{c}3.0 \\
(2.0-4.1)\end{array}$ & 0.91 & & $\begin{array}{c}0.2 \\
(-0.3-0.7)\end{array}$ & $\begin{array}{c}0.4 \\
(0.1-0.7)\end{array}$ & $\begin{array}{c}0.1 \\
(-0.4-0.7)\end{array}$ & 0.93 & \\
\hline Exercisers & $\begin{array}{c}3.0 \\
(2.3-3.7)\end{array}$ & $\begin{array}{c}2.2 \\
(1.6-2.7)\end{array}$ & $1.6(0.4-2.7)$ & $0.01 *$ & & $\begin{array}{c}0.4 \\
(-0.1-0.9)\end{array}$ & $0.4(0.1-0.7)$ & $0(-0.7-0.8)$ & 0.45 & \\
\hline Wine & & & & & 0.48 & & & & & $0.03 *$ \\
\hline Non-exercisers & $\begin{array}{c}2.3 \\
(1.2-3.4)\end{array}$ & $\begin{array}{c}3.4 \\
(2.6-4.1)\end{array}$ & $\begin{array}{c}4.4 \\
(3.0-5.8)\end{array}$ & $0.02 *$ & & $\begin{array}{c}6.0 \\
(3.6-8.3)\end{array}$ & $\begin{array}{c}5.0 \\
(3.6-6.4)\end{array}$ & $\begin{array}{c}6.6 \\
(4.0-9.3)\end{array}$ & 0.71 & \\
\hline Exercisers & $4.2(2.8-5.6)$ & $5.3(4.2-6.3)$ & $\begin{array}{c}4.5 \\
(2.2-6.9) \\
\end{array}$ & 0.51 & & $\begin{array}{c}8.7 \\
(6.3-11.1) \\
\end{array}$ & $\begin{array}{c}4.6 \\
(3.1-6.2) \\
\end{array}$ & $4.4(0.7-8.1)$ & $0.01 *$ & \\
\hline
\end{tabular}

NS, not significant; SRPS, self-reported psychological stress; ${ }^{*} \mathrm{p}<0.05 ;{ }^{1}$ Multi-adjusted linear regression analyses were adjusted for age, body mass index, exercise, smoking, energy, alcohol intake, history of disease (hypertension and diabetes mellitus) and sleeping time. Drinking, exercise and history of disease were assumed to be dichotomous variables (non-drinker, non-exercisers or no history of hypertension or diabetes mellitus=0; drinker, habitual exercisers or having a history of hypertension or diabetes mellitus=1). SRPS levels and smoking were assumed to be a trichotomous variable (low or never $=0$; moderate or past $=1$; high or current $=2$ ). Dietary intake of 58 food items was estimated from a food frequency questionnaire; ${ }^{2}$ Values are shown as the adjusted means (standard error); ${ }^{3}$ Non-exercisers $($ men $=2,918$; women $=1,485) ;{ }^{4}$ Exercisers $(\mathrm{men}=2,669$; women $=1,233)$.

Table 3. Interactions between SRPS and Habitual Exercise Level in the Dietary Intake of Energy and Food Types in Middle-aged Japanese men and Women ${ }^{1}$

\begin{tabular}{|c|c|c|c|c|c|c|c|c|c|c|}
\hline \multirow{3}{*}{$\begin{array}{c}\text { (g or mg/1000kcal/ } \\
\text { day) }\end{array}$} & \multicolumn{5}{|c|}{ Men $(n=5,587)$} & \multicolumn{5}{|c|}{ Women $(\mathrm{n}=2,718)$} \\
\hline & \multicolumn{3}{|c|}{ SRPS } & \multirow[b]{2}{*}{$\begin{array}{l}\mathrm{p} \text { for } \\
\text { trend }\end{array}$} & \multirow[b]{2}{*}{$\begin{array}{l}\mathrm{p} \text { for } \\
\text { int }\end{array}$} & \multicolumn{3}{|c|}{ SRPS } & \multirow[b]{2}{*}{$\begin{array}{l}\mathrm{p} \text { for } \\
\text { trend }\end{array}$} & \multirow[b]{2}{*}{$\begin{array}{l}\mathrm{p} \text { for } \\
\text { int }\end{array}$} \\
\hline & $\begin{array}{c}\text { Low }^{2} \\
(n=1,558)\end{array}$ & $\begin{array}{l}\text { Moderate }^{2} \\
(\mathrm{n}=3,247)\end{array}$ & $\begin{array}{c}\mathrm{High}^{2} \\
(\mathrm{n}=782)\end{array}$ & & & $\begin{array}{c}\mathrm{Low}^{2} \\
(\mathrm{n}=641)\end{array}$ & $\begin{array}{l}\text { Moderate }^{2} \\
(\mathrm{n}=1,691)\end{array}$ & $\begin{array}{c}\operatorname{High}^{2} \\
(\mathrm{n}=386)\end{array}$ & & \\
\hline Energy $^{a}$ & & & & & 0.47 & & & & & 0.64 \\
\hline Non-exercisers ${ }^{3}$ & $\begin{array}{c}2088 \\
(2054-2122)\end{array}$ & $\begin{array}{c}2136 \\
(2113-2158)\end{array}$ & $\begin{array}{c}2134 \\
(2091-2176)\end{array}$ & 0.66 & & $\begin{array}{c}1570 \\
(1535-1605)\end{array}$ & $\begin{array}{c}1588 \\
(1567-1608)\end{array}$ & $\begin{array}{c}1597 \\
(1558-1636)\end{array}$ & 0.27 & \\
\hline Exercisers $^{4}$ & $\begin{array}{c}2166(2134- \\
2198)\end{array}$ & $\begin{array}{c}2180 \\
(2156-2204)\end{array}$ & $\begin{array}{c}2176 \\
(2124-2229)\end{array}$ & 0.06 & & $\begin{array}{c}1597 \\
(1561-1632)\end{array}$ & $\begin{array}{c}1603 \\
(1580-1626)\end{array}$ & $\begin{array}{c}1579 \\
(1524-1634)\end{array}$ & 0.69 & \\
\hline Protein & & & & & 0.36 & & & & & 0.09 \\
\hline Non-exercisers & $\begin{array}{c}29.3 \\
(29.0-29.6)\end{array}$ & $\begin{array}{c}29.6 \\
(29.4-29.8)\end{array}$ & $\begin{array}{c}29.4 \\
(29.0-29.8)\end{array}$ & 0.43 & & $\begin{array}{c}32.7 \\
(32.3-33.2)\end{array}$ & $\begin{array}{c}33.0 \\
(32.7-33.3)\end{array}$ & $\begin{array}{c}33.2 \\
(32.6-33.7)\end{array}$ & 0.22 & \\
\hline Exercisers & $\begin{array}{c}30.4 \\
(30.1-30.7)\end{array}$ & $\begin{array}{c}30.3(30.1- \\
30.6)\end{array}$ & $\begin{array}{c}30.2 \\
(29.7-30.6)\end{array}$ & 0.63 & & $\begin{array}{c}34.1 \\
(33.6-34.7)\end{array}$ & $\begin{array}{c}33.9 \\
(33.6-34.2)\end{array}$ & $\begin{array}{c}33.0 \\
(32.2-33.8)\end{array}$ & $0.03^{*}$ & \\
\hline Animal Protein & & & & & 0.50 & & & & & 0.51 \\
\hline Non-exercisers & $\begin{array}{c}14.3 \\
(14.0-14.7)\end{array}$ & $\begin{array}{c}14.6 \\
(14.4-14.9)\end{array}$ & $\begin{array}{c}14.5 \\
(14.1-14.9)\end{array}$ & 0.88 & & $\begin{array}{c}16.3 \\
(15.8-16.9)\end{array}$ & $\begin{array}{c}16.6 \\
(16.3-16.9)\end{array}$ & $\begin{array}{c}16.7 \\
(16.1-17.3)\end{array}$ & 0.36 & \\
\hline Exercisers & $\begin{array}{c}15.2 \\
(14.9-15.5)\end{array}$ & $\begin{array}{c}15.3 \\
(15.0-15.5)\end{array}$ & $\begin{array}{c}15.1 \\
(14.6-15.6)\end{array}$ & 0.40 & & $\begin{array}{c}17.5 \\
(17.0-18.1)\end{array}$ & $\begin{array}{c}17.2 \\
(16.9-17.6)\end{array}$ & $\begin{array}{c}16.5 \\
(15.6-17.3)\end{array}$ & $0.03 *$ & \\
\hline Vegetable Protein & & & & & 0.73 & & & & & $0.01^{*}$ \\
\hline Non-exercisers & $\begin{array}{c}15.0 \\
(14.9-15.1)\end{array}$ & $\begin{array}{c}14.9 \\
(14.8-15.0)\end{array}$ & $\begin{array}{c}14.9 \\
(14.8-15.1)\end{array}$ & 0.37 & & $\begin{array}{c}16.4 \\
(16.2-16.6)\end{array}$ & $\begin{array}{c}16.4 \\
(16.3-16.5)\end{array}$ & $\begin{array}{c}16.4 \\
(16.2-16.7)\end{array}$ & 0.73 & \\
\hline Exercisers & $\begin{array}{c}15.2 \\
(15.1-15.3)\end{array}$ & $\begin{array}{c}15.1 \\
(15.0-15.2)\end{array}$ & $\begin{array}{c}15.1 \\
(14.9-15.3)\end{array}$ & 0.22 & & $\begin{array}{c}16.6 \\
(16.4-16.8)\end{array}$ & $\begin{array}{c}16.7 \\
(16.5-16.8)\end{array}$ & $\begin{array}{c}16.6 \\
(16.2-16.9)\end{array}$ & 0.07 & \\
\hline Fat & & & & & 0.16 & & & & & 0.77 \\
\hline Non-exercisers & $\begin{array}{c}23.1 \\
(22.7-23.5)\end{array}$ & $\begin{array}{c}23.2 \\
(22.9-23.5)\end{array}$ & $\begin{array}{c}23.6 \\
(23.1-24.1)\end{array}$ & 0.12 & & $\begin{array}{c}28.3 \\
(27.7-28.9)\end{array}$ & $\begin{array}{c}28.4 \\
(28.0-28.7)\end{array}$ & $\begin{array}{c}28.1 \\
(27.5-28.8)\end{array}$ & 0.74 & \\
\hline Exercisers & $\begin{array}{c}24.1 \\
(23.8-24.5)\end{array}$ & $\begin{array}{c}24.0 \\
(23.8-24.3)\end{array}$ & $\begin{array}{c}24.0 \\
(23.4-24.6)\end{array}$ & 0.63 & & $\begin{array}{c}28.8 \\
(28.2-29.4)\end{array}$ & $\begin{array}{c}28.7 \\
(28.3-29.1)\end{array}$ & $\begin{array}{c}27.7 \\
(26.8-28.6)\end{array}$ & 0.09 & \\
\hline Animal Fat & & & & & 0.11 & & & & & 0.41 \\
\hline Non-exercisers & $\begin{array}{c}10.0 \\
(9.8-10.3)\end{array}$ & $\begin{array}{c}10.3 \\
(10.1-10.4)\end{array}$ & $\begin{array}{c}10.4 \\
(10.1-10.7)\end{array}$ & 0.05 & & $\begin{array}{c}12.0 \\
(11.6-12.4)\end{array}$ & $\begin{array}{c}12.1 \\
(11.9-12.4)\end{array}$ & $\begin{array}{c}12.0 \\
(11.6-12.5)\end{array}$ & 0.94 & \\
\hline Exercisers & $\begin{array}{c}10.6 \\
(10.4-10.9)\end{array}$ & $\begin{array}{c}10.6 \\
(10.5-10.8)\end{array}$ & $\begin{array}{c}10.5 \\
(10.1-10.9)\end{array}$ & 0.71 & & $\begin{array}{c}12.4 \\
(12.0-12.8)\end{array}$ & $\begin{array}{c}12.2(11.9- \\
12.4)\end{array}$ & $\begin{array}{c}11.7 \\
(11.1-12.4)\end{array}$ & 0.07 & \\
\hline Vegetable Fat & & & & & 0.55 & & & & & 0.31 \\
\hline Non-exercisers & $\begin{array}{c}13.0 \\
(12.8-13.3)\end{array}$ & $\begin{array}{c}12.9 \\
(12.8-13.1)\end{array}$ & $\begin{array}{c}13.2 \\
(12.9-13.5)\end{array}$ & 0.54 & & $\begin{array}{c}16.3 \\
(15.9-16.7)\end{array}$ & $\begin{array}{c}16.2 \\
(16.0-16.5)\end{array}$ & $\begin{array}{c}16.1 \\
(15.6-16.5)\end{array}$ & 0.41 & \\
\hline Exercisers & $\begin{array}{c}13.5 \\
(13.2-13.7)\end{array}$ & $\begin{array}{c}13.4 \\
(13.2-13.6)\end{array}$ & $\begin{array}{c}13.5 \\
(13.1-13.9)\end{array}$ & 0.77 & & $\begin{array}{c}16.4 \\
(16.0-16.9)\end{array}$ & $\begin{array}{c}16.5 \\
(16.2-16.8)\end{array}$ & $\begin{array}{c}16.0 \\
(15.3-16.7)\end{array}$ & 0.46 & \\
\hline Carbohydrate & & & & & 0.16 & & & & & 0.82 \\
\hline Non-exercisers & $\begin{array}{c}138.3 \\
(137.2-139.4)\end{array}$ & $\begin{array}{c}137.7 \\
(137.0-138.4)\end{array}$ & $\begin{array}{c}136.9 \\
(135.6- \\
138.3)\end{array}$ & 0.13 & & $\begin{array}{c}143.1 \\
(141.5-144.6)\end{array}$ & $\begin{array}{c}142.6 \\
(141.7-143.5)\end{array}$ & $\begin{array}{c}143.0 \\
(141.3-144.7)\end{array}$ & 0.84 & \\
\hline Exercisers & $\begin{array}{c}135.7 \\
(134.7-136.6)\end{array}$ & $\begin{array}{c}135.9(135.2- \\
136.6)\end{array}$ & $\begin{array}{c}136.1 \\
(134.5- \\
137.7) \\
\end{array}$ & 0.59 & & $\begin{array}{c}141.1(139.6- \\
142.6)\end{array}$ & $\begin{array}{c}141.6(140.6- \\
142.5)\end{array}$ & $\begin{array}{c}144.3(141.9- \\
146.6)\end{array}$ & 0.05 & \\
\hline
\end{tabular}


DOI:http://dx.doi.org/10.7314/APJCP.2016.17.4.2007

Impact of Interaction of Psychological Stress with Habitual Exercise on Dietary Intake

Table 3 (continued). Interactions between SRPS and Habitual Exercise Level in the Dietary Intake of Energy and Food Types in Middle-aged Japanese men and Women ${ }^{1}$

\begin{tabular}{|c|c|c|c|c|c|c|c|c|c|c|}
\hline \multirow{3}{*}{$\begin{array}{c}\text { (g/1000 kcal/ } \\
\text { day) }\end{array}$} & \multicolumn{5}{|c|}{$\operatorname{Men}(n=5,587)$} & \multicolumn{5}{|c|}{ Women $(n=2,718)$} \\
\hline & \multicolumn{3}{|c|}{ SRPS } & \multirow[b]{2}{*}{$\begin{array}{l}\mathrm{p} \text { for } \\
\text { trend }\end{array}$} & \multirow[b]{2}{*}{$\begin{array}{l}\mathrm{p} \text { for } \\
\text { int }\end{array}$} & \multicolumn{3}{|c|}{ SRPS } & \multirow[b]{2}{*}{$\begin{array}{l}\mathrm{p} \text { for } \\
\text { trend }\end{array}$} & \multirow[b]{2}{*}{$\begin{array}{c}\mathrm{p} \text { for } \\
\text { int }\end{array}$} \\
\hline & $\begin{array}{c}\text { Low }^{2} \\
(n=1,558) \\
\end{array}$ & $\begin{array}{l}\text { Moderate }^{2} \\
(\mathrm{n}=3,247)\end{array}$ & $\begin{array}{c}\text { High }^{2} \\
(\mathrm{n}=782)\end{array}$ & & & $\begin{array}{c}\text { Low }^{2} \\
(n=641)\end{array}$ & $\begin{array}{l}\text { Moderate }^{2} \\
(\mathrm{n}=1,691)\end{array}$ & $\begin{array}{c}\text { High }^{2} \\
(\mathrm{n}=386)\end{array}$ & & \\
\hline Alcohol & & & & & 0.98 & & & & & 0.10 \\
\hline Non-exercisers & $\begin{array}{c}15.6 \\
(14.7-16.5)\end{array}$ & $\begin{array}{c}14.6 \\
(14.0-15.2)\end{array}$ & $\begin{array}{c}15.2 \\
(14.0-16.3)\end{array}$ & 0.39 & & $4.3(3.5-5.1)$ & $4.1(3.6-4.6)$ & $4.7(3.8-5.7)$ & 0.50 & \\
\hline Exercisers & $\begin{array}{c}15.2 \\
(14.4-16.0)\end{array}$ & $\begin{array}{c}14.5 \\
(13.9-15.1)\end{array}$ & $\begin{array}{c}15.0 \\
(13.7-16.3)\end{array}$ & 0.45 & & $4.4(3.6-5.1)$ & $4.2(3.7-4.7)$ & $4.3(3.1-5.4)$ & 0.74 & \\
\hline SFA & & & & & 0.10 & & & & & 0.86 \\
\hline Non-exercisers & $\begin{array}{c}5.5 \\
(5.4-5.6)\end{array}$ & $\begin{array}{c}5.6 \\
(5.5-5.7)\end{array}$ & $\begin{array}{c}5.7 \\
(5.6-5.9)\end{array}$ & $0.03 *$ & & $7.0(6.8-7.2)$ & $7.1(7.0-7.2)$ & $7.0(6.8-7.2)$ & 0.99 & \\
\hline Exercisers & $\begin{array}{c}5.8 \\
(5.7-5.9)\end{array}$ & $\begin{array}{c}5.8 \\
(5.7-5.9)\end{array}$ & $\begin{array}{c}5.8 \\
(5.6-6.0)\end{array}$ & 0.85 & & $7.1(6.9-7.2)$ & $7.1(6.9-7.2)$ & $7.0(6.7-7.2)$ & 0.48 & \\
\hline MUFA & & & & & 0.19 & & & & & 0.75 \\
\hline Non-exercisers & $\begin{array}{c}8.2 \\
(8.0-8.4)\end{array}$ & $\begin{array}{c}8.2 \\
(8.1-8.3)\end{array}$ & $\begin{array}{c}8.4 \\
(8.2-8.6)\end{array}$ & 0.12 & & $\begin{array}{c}10.0( \\
9.8-10.2)\end{array}$ & $\begin{array}{c}10.0 \\
(9.9-10.1)\end{array}$ & $9.9(9.7-10.2)$ & 0.62 & \\
\hline Exercisers & $\begin{array}{c}8.5 \\
(8.4-8.6)\end{array}$ & $\begin{array}{c}8.5 \\
(8.4-8.6)\end{array}$ & $\begin{array}{c}8.5 \\
(8.2-8.7)\end{array}$ & 0.74 & & $\begin{array}{c}10.1 \\
(9.9-10.4)\end{array}$ & $\begin{array}{c}10.1 \\
(9.9-10.2)\end{array}$ & $9.7(9.4-10.1)$ & 0.07 & \\
\hline PUFA & & & & & 0.40 & & & & & 0.51 \\
\hline Non-exercisers & $\begin{array}{c}6.3 \\
(6.2-6.4)\end{array}$ & $\begin{array}{c}6.3 \\
(6.2-6.4)\end{array}$ & $\begin{array}{c}6.4 \\
(6.2-6.5)\end{array}$ & 0.61 & & $7.5(7.3-7.7)$ & $7.5(7.4-7.6)$ & $7.4(7.2-7.6)$ & 0.73 & \\
\hline Exercisers & $\begin{array}{c}6.6 \\
(6.5-6.7)\end{array}$ & $\begin{array}{c}6.5 \\
(6.5-6.6)\end{array}$ & $\begin{array}{c}6.5 \\
(6.3-6.7)\end{array}$ & 0.44 & & $7.7(7.5-7.9)$ & $7.7(7.5-7.8)$ & $7.3(7.0-7.6)$ & 0.06 & \\
\hline n-3 PUFA & & & & & 0.32 & & & & & 0.47 \\
\hline Non-exercisers & $\begin{array}{c}1.3 \\
(1.3-1.3)\end{array}$ & $\begin{array}{c}1.3 \\
(1.3-1.3)\end{array}$ & $\begin{array}{c}1.3 \\
(1.3-1.4)\end{array}$ & 0.62 & & $1.5(1.5-1.6)$ & $1.5(1.5-1.6)$ & $1.5(1.5-1.6)$ & 0.60 & \\
\hline Exercisers & $\begin{array}{c}1.4 \\
(1.4-1.4)\end{array}$ & $\begin{array}{c}1.4 \\
(1.3-1.4)\end{array}$ & $\begin{array}{c}1.4 \\
(1.3-1.4)\end{array}$ & 0.42 & & $1.6(1.6-1.6)$ & $1.6(1.6-1.6)$ & $1.5(1.4-1.6)$ & 0.10 & \\
\hline n-6 PUFA & & & & & 0.49 & & & & & 0.85 \\
\hline Non-exercisers & $\begin{array}{c}5.3 \\
(5.2-5.4)\end{array}$ & $\begin{array}{c}5.3 \\
(5.2-5.3)\end{array}$ & $\begin{array}{c}5.3 \\
(5.2-5.5)\end{array}$ & 0.78 & & $6.3(6.2-6.5)$ & $6.3(6.2-6.4)$ & $6.3(6.1-6.4)$ & 0.58 & \\
\hline Exercisers & $\begin{array}{c}5.5 \\
(5.4-5.6)\end{array}$ & $\begin{array}{c}5.5 \\
(5.4-5.5)\end{array}$ & $\begin{array}{c}5.4 \\
(5.3-5.6)\end{array}$ & 0.48 & & $6.5(6.3-6.6)$ & $6.4(6.3-6.5)$ & $6.1(5.9-6.4)$ & 0.06 & \\
\hline n-6 PUFA/n-3 PUFA & & & & & 0.80 & & & & & 0.24 \\
\hline Non-exercisers & $\begin{array}{c}4.2 \\
(4.1-4.2)\end{array}$ & $\begin{array}{c}4.2 \\
(4.2-4.2)\end{array}$ & $\begin{array}{c}4.2 \\
(4.1-4.2)\end{array}$ & 0.83 & & $4.2(4.1-4.3)$ & $4.2(4.2-4.2)$ & $4.2(4.1-4.3)$ & 0.77 & \\
\hline Exercisers & $\begin{array}{c}4.1 \\
(4.0-4.1)\end{array}$ & $\begin{array}{c}4.1 \\
(4.1-4.1)\end{array}$ & $\begin{array}{c}4.0 \\
(4.0-4.1)\end{array}$ & 0.71 & & $4.1(4.1-4.2)$ & $4.1(4.0-4.1)$ & $4.1(4.0-4.3)$ & 0.84 & \\
\hline Cholesterol & & & & & 0.54 & & & & & 0.36 \\
\hline Non-exercisers & $\begin{array}{c}134 \\
(130-138)\end{array}$ & $\begin{array}{c}135 \\
(133-137)\end{array}$ & $\begin{array}{c}137 \\
(132-142)\end{array}$ & 0.27 & & $\begin{array}{c}156 \\
(151-162)\end{array}$ & $157(154-161)$ & $\begin{array}{c}158(152- \\
164)\end{array}$ & 0.69 & \\
\hline Exercisers & $\begin{array}{c}138 \\
(135-142)\end{array}$ & $\begin{array}{c}140 \\
(138-143)\end{array}$ & $\begin{array}{c}139 \\
(131-143)\end{array}$ & 0.97 & & $\begin{array}{c}164 \\
(158-169)\end{array}$ & $160(156-163)$ & $\begin{array}{c}161(152- \\
170)\end{array}$ & 0.29 & \\
\hline Soluble dietary fiber & & & & & 0.37 & & & & & \\
\hline Non-exercisers & $1.1(1.1-1.1)$ & $1.1(1.1-1.1)$ & $1.1(1.1-1.1)$ & 0.95 & & $1.4(1.4-1.5)$ & $1.4(1.4-1.5)$ & $1.4(1.4-1.5)$ & 0.87 & \\
\hline Exercisers & $1.2(1.2-1.2)$ & $1.2(1.2-1.2)$ & $1.2(1.2-1.2)$ & 0.28 & & $1.6(1.5-1.6)$ & $1.6(1.5-1.6)$ & $1.5(1.4-1.6)$ & 0.15 & \\
\hline Insoluble dietary fiber & & & & & 0.16 & & & & & $<0.01 *$ \\
\hline Non-exercisers & $3.5(3.5-3.6)$ & $3.5(3.4-3.5)$ & $3.5(3.4-3.6)$ & 0.27 & & $4.4(4.3-4.5)$ & $4.3(4.3-4.4)$ & $4.3(4.2-4.5)$ & 0.35 & \\
\hline Exercisers & $3.8(3.8-3.9)$ & $3.7(3.7-3.8)$ & $3.7(3.6-3.8)$ & $<0.01 *$ & & $4.7(4.6-4.8)$ & $4.6(4.6-4.7)$ & $4.5(4.3-4.6)$ & 0.09 & \\
\hline Total dietary fiber & & & & & 0.28 & & & & & $0.001 *$ \\
\hline Non-exercisers & $4.8(4.7-4.9)$ & $4.7(4.7-4.8)$ & $4.7(4.6-4.9)$ & 0.31 & & $6.1(6.0-6.3)$ & $6.0(5.9-6.1)$ & $6.0(5.9-6.2)$ & 0.47 & \\
\hline Exercisers & $5.2(5.1-5.3)$ & $5.1(5.0-5.2)$ & $5.1(4.9-5.2)$ & $0.02 *$ & & $6.6(6.4-6.7)$ & $6.5(6.4-6.6)$ & $6.2(6.0-6.5)$ & 0.10 & \\
\hline Daizein & & & & & 0.83 & & & & & $0.04 *$ \\
\hline Non-exercisers & $4.3(4.1-4.5)$ & $4.2(4.1-4.3)$ & $4.2(3.9-4.4)$ & 0.33 & & $5.6(5.2-5.9)$ & $5.5(5.3-5.7)$ & $5.7(5.3-6.1)$ & 0.75 & \\
\hline Exercisers & $5.1(4.9-5.3)$ & $5.0(4.9-5.2)$ & $5.0(4.6-5.3)$ & 0.40 & & $6.3(5.9-6.6)$ & $6.2(5.9-6.4)$ & $5.8(5.3-6.4)$ & 0.26 & \\
\hline Genistein & & & & & 0.83 & & & & & $0.04 *$ \\
\hline Non-exercisers & $7.3(7.0-7.7)$ & $7.1(6.9-7.4)$ & $7.1(6.6-7.5)$ & 0.33 & & $9.5(8.9-10.1)$ & $9.4(9.0-9.7)$ & $9.6(8.9-10.3)$ & 0.75 & \\
\hline Exercisers & $8.7(8.3-9.0)$ & $8.5(8.3-8.8)$ & $8.4(7.8-9.0)$ & 0.40 & & $\begin{array}{c}10.6 \\
(10.0-11.2)\end{array}$ & $\begin{array}{c}10.4 \\
(10.0-10.8)\end{array}$ & $9.9(8.9-10.9)$ & 0.25 & \\
\hline Retinolb & & & & & 0.99 & & & & & 0.29 \\
\hline Non-exercisers & $179(169-188)$ & $178(172-185)$ & $\begin{array}{c}167(155- \\
179)\end{array}$ & 0.18 & & $\begin{array}{l}186(171- \\
200)\end{array}$ & $190(181-198)$ & $\begin{array}{c}196 \\
(180-212)\end{array}$ & 0.40 & \\
\hline Exercisers & $192(183-202)$ & $190(184-197)$ & $\begin{array}{c}180(165- \\
195)\end{array}$ & 0.23 & & $\begin{array}{l}194(180- \\
207)\end{array}$ & $196(187-204)$ & $\begin{array}{c}198 \\
(178-219)\end{array}$ & 0.77 & \\
\hline Carotene $^{b}$ & & & & & 0.43 & & & & & $0.01 *$ \\
\hline Non-exercisers & $\begin{array}{c}966 \\
(926-1007)\end{array}$ & $938(912-965)$ & $\begin{array}{c}926(875- \\
976)\end{array}$ & 0.23 & & $\begin{array}{c}1512 \\
(1429-1597)\end{array}$ & $\begin{array}{c}1483 \\
(1434-1532)\end{array}$ & $\begin{array}{c}1451 \\
(1357-1545)\end{array}$ & 0.34 & \\
\hline Exercisers & $\begin{array}{c}1138 \\
(1095-1180)\end{array}$ & $\begin{array}{c}1084 \\
(1053-1115)\end{array}$ & $\begin{array}{c}1072 \\
(1003-1141)\end{array}$ & 0.05 & & $\begin{array}{c}1680(1586- \\
1774)\end{array}$ & $\begin{array}{c}1661 \\
(1601-1721)\end{array}$ & $\begin{array}{c}1573 \\
(1428-1718)\end{array}$ & 0.25 & \\
\hline Retinol equivalent $\mathrm{t}^{\mathrm{b}}$ & & & & & 0.66 & & & & & $0.01 *$ \\
\hline Non-exercisers & $338(326-351)$ & $\begin{array}{c}333.4 \\
(326-342)\end{array}$ & $\begin{array}{c}320(305- \\
336)\end{array}$ & 0.10 & & $\begin{array}{c}436(416- \\
457)\end{array}$ & 435 (423-447) & $\begin{array}{c}436 \\
(413-459)\end{array}$ & 0.92 & \\
\hline Exercisers & $381(369-393)$ & $370(361-379)$ & $\begin{array}{c}358(338- \\
377)\end{array}$ & $0.04 *$ & & $\begin{array}{c}472(451- \\
493)\end{array}$ & $471(457-484)$ & $\begin{array}{c}459 \\
(426-491)\end{array}$ & 0.51 & \\
\hline
\end{tabular}


Table 3 (continued). Interactions between SRPS and Habitual Exercise Level in the Dietary Intake of Energy and Food Types in Middle-aged Japanese men and Women ${ }^{1}$

\begin{tabular}{|c|c|c|c|c|c|c|c|c|c|c|}
\hline \multirow{3}{*}{$\begin{array}{c}\text { (g or mg/1000kcal/ } \\
\text { day) }\end{array}$} & \multicolumn{5}{|c|}{ Men $(n=5,587)$} & \multicolumn{5}{|c|}{ Women $(n=2,718)$} \\
\hline & \multicolumn{3}{|c|}{ SRPS } & \multirow[b]{2}{*}{$\begin{array}{l}\mathrm{p} \text { for } \\
\text { trend }\end{array}$} & \multirow[b]{2}{*}{$\begin{array}{c}\mathrm{p} \text { for } \\
\text { int }\end{array}$} & \multicolumn{3}{|c|}{ SRPS } & \multirow[b]{2}{*}{$\begin{array}{l}\mathrm{p} \text { for } \\
\text { trend }\end{array}$} & \multirow[b]{2}{*}{$\begin{array}{l}\mathrm{p} \text { for } \\
\text { int }\end{array}$} \\
\hline & $\begin{array}{c}\text { Low }^{2} \\
(\mathrm{n}=1,558)\end{array}$ & $\begin{array}{l}\text { Moderate }^{2} \\
(\mathrm{n}=3,247)\end{array}$ & $\begin{array}{c}\mathrm{High}^{2} \\
(\mathrm{n}=782)\end{array}$ & & & $\begin{array}{c}\text { Low }^{2} \\
(\mathrm{n}=641)\end{array}$ & $\begin{array}{l}\text { Moderate }^{2} \\
(\mathrm{n}=1,691)\end{array}$ & $\begin{array}{c}\mathrm{High}^{2} \\
(\mathrm{n}=386)\end{array}$ & & \\
\hline Vitamin $\mathrm{D}^{\mathrm{b}}$ & & & & & 0.34 & & & & & 0.19 \\
\hline Non-exercisers & $3.8(3.7-4.0)$ & $3.9(3.8-4.0)$ & $3.9(3.7-4.1)$ & 0.60 & & $4.3(4.1-4.5)$ & $4.3(4.2-4.4)$ & $4.4(4.2-4.7)$ & 0.65 & \\
\hline Exercisers & $4.3(4.1-4.4)$ & $4.2(4.1-4.4)$ & $4.2(3.9-4.4)$ & 0.46 & & $4.8(4.5-5.0)$ & $4.7(4.6-4.9)$ & $4.5(4.1-4.9)$ & 0.20 & \\
\hline Vitamin E & & & & & 0.42 & & & & & 0.18 \\
\hline Non-exercisers & $3.6(3.6-3.7)$ & $3.6(3.6-3.7)$ & $3.6(3.5-3.7)$ & 0.86 & & $4.5(4.4-4.7)$ & $4.5(4.5-4.6)$ & $4.5(4.4-4.6)$ & 0.50 & \\
\hline Exercisers & $3.9(3.8-3.9)$ & $3.8(3.8-3.9)$ & $3.8(3.7-3.9)$ & 0.23 & & $4.7(4.6-4.9)$ & $4.7(4.6-4.8)$ & $4.5(4.3-4.7)$ & $0.03 *$ & \\
\hline Vitamin $\mathrm{K}^{\mathrm{b}}$ & & & & & 0.34 & & & & & 0.10 \\
\hline Non-exercisers & $169(164-174)$ & $168(164-171)$ & $\begin{array}{c}168 \\
(161-175)\end{array}$ & 0.71 & & $\begin{array}{c}221 \\
(212-230)\end{array}$ & $223(217-228)$ & $\begin{array}{c}229 \\
(219-240)\end{array}$ & 0.25 & \\
\hline Exercisers & 199 (193-204) & $190(185-194)$ & $\begin{array}{c}195 \\
(186-205)\end{array}$ & 0.17 & & $\begin{array}{c}243 \\
(232-253)\end{array}$ & $242(235-248)$ & $\begin{array}{c}226 \\
(211-241)\end{array}$ & 0.15 & \\
\hline Vitamin B1 & & & & & 0.45 & & & & & 0.13 \\
\hline Non-exercisers & $\begin{array}{c}0.30 \\
(0.30-0.31)\end{array}$ & $\begin{array}{c}0.31 \\
(0.30-0.31)\end{array}$ & $\begin{array}{c}0.30 \\
(0.30-0.30)\end{array}$ & 0.87 & & $\begin{array}{c}0.38 \\
(0.37-0.38)\end{array}$ & $\begin{array}{c}0.38 \\
(0.37-0.38)\end{array}$ & $\begin{array}{c}0.38 \\
(0.37-0.39)\end{array}$ & 0.70 & \\
\hline Exercisers & $\begin{array}{c}0.32 \\
(0.32-0.33)\end{array}$ & $\begin{array}{c}0.32 \\
(0.32-0.32)\end{array}$ & $\begin{array}{c}0.32 \\
(0.31-0.32)\end{array}$ & 0.30 & & $\begin{array}{c}0.40 \\
(0.39-0.41)\end{array}$ & $\begin{array}{c}0.39 \\
(0.39-0.40)\end{array}$ & $\begin{array}{c}0.38 \\
(0.37-0.39)\end{array}$ & $<0.001^{*}$ & \\
\hline Vitamin B2 & & & & & 0.67 & & & & & $0.02 *$ \\
\hline Non-exercisers & $\begin{array}{c}0.55 \\
(0.54-0.56)\end{array}$ & $\begin{array}{c}0.55 \\
(0.54-0.55)\end{array}$ & $\begin{array}{c}0.54 \\
(0.53-0.55)\end{array}$ & 0.57 & & $\begin{array}{c}0.64 \\
(0.63-0.66)\end{array}$ & $\begin{array}{c}0.66 \\
(0.65-0.66)\end{array}$ & $\begin{array}{c}0.67 \\
(0.65-0.68)\end{array}$ & $0.03 *$ & \\
\hline Exercisers & $\begin{array}{c}0.59 \\
(0.58-0.60)\end{array}$ & $\begin{array}{c}0.58 \\
(0.58-0.59)\end{array}$ & $\begin{array}{c}0.58 \\
(0.57-0.60)\end{array}$ & 0.28 & & $\begin{array}{c}0.69 \\
(0.68-0.71)\end{array}$ & $\begin{array}{c}0.69 \\
(0.68-0.70)\end{array}$ & $\begin{array}{c}0.67 \\
(0.65-0.69)\end{array}$ & 0.16 & \\
\hline Niacin & & & & & 0.70 & & & & & 0.19 \\
\hline Non-exercisers & $7.6(7.5-7.8)$ & $7.6(7.5-7.7)$ & $7.5(7.4-7.7)$ & 0.37 & & $7.8(7.6-8.0)$ & $7.9(7.8-8.0)$ & $8.0(7.8-8.2)$ & 0.15 & \\
\hline Exercisers & $7.8(7.6-7.9)$ & $7.8(7.7-7.9)$ & $7.7(7.5-7.9)$ & 0.71 & & $8.2(8.0-8.4)$ & $8.2(8.1-8.4)$ & $7.8(7.5-8.1)$ & 0.07 & \\
\hline Vitamin B6 & & & & & 0.87 & & & & & 0.06 \\
\hline Non-exercisers & $\begin{array}{c}0.51(0.50- \\
0.52)\end{array}$ & $\begin{array}{c}0.51 \\
(0.50-0.51)\end{array}$ & $\begin{array}{c}0.50 \\
(0.49-0.52)\end{array}$ & 0.27 & & $\begin{array}{c}0.57 \\
(0.56-0.59)\end{array}$ & $\begin{array}{c}0.57 \\
(0.56-0.58)\end{array}$ & $\begin{array}{c}0.58 \\
(0.57-0.60)\end{array}$ & 0.44 & \\
\hline Exercisers & $\begin{array}{c}0.54(0.54- \\
0.55)\end{array}$ & $\begin{array}{c}0.54 \\
(0.53-0.54)\end{array}$ & $\begin{array}{c}0.54 \\
(0.53-0.55)\end{array}$ & 0.39 & & $\begin{array}{c}0.61 \\
(0.60-0.62)\end{array}$ & $\begin{array}{c}0.61 \\
(0.60-0.62)\end{array}$ & $\begin{array}{c}0.58 \\
(0.56-0.60)\end{array}$ & $0.02 *$ & \\
\hline Vitamin B12 ${ }^{\mathrm{b}}$ & & & & & 0.95 & & & & & 0.20 \\
\hline Non-exercisers & $3.4(3.3-3.5)$ & $3.5(3.4-3.6)$ & $3.3(3.2-3.5)$ & 0.79 & & $3.5(3.3-3.7)$ & $3.5(3.4-3.6)$ & $3.6(3.4-3.8)$ & 0.45 & \\
\hline Exercisers & $3.7(3.6-3.8)$ & $3.7(3.6-3.8)$ & $3.6(3.4-3.8)$ & 0.72 & & $3.7(3.5-3.9)$ & $3.8(3.7-3.9)$ & $3.6(3.4-3.9)$ & 0.79 & \\
\hline Folate $^{\mathrm{b}}$ & & & & & 0.66 & & & & & 0.14 \\
\hline Non-exercisers & $138(135-141)$ & $136(135-138)$ & $\begin{array}{c}134 \\
(130-137)\end{array}$ & 0.07 & & $\begin{array}{c}169 \\
(164-174)\end{array}$ & $169(166-172)$ & $\begin{array}{c}173 \\
(167-178)\end{array}$ & 0.34 & \\
\hline Exercisers & $151(148-153)$ & $146(144-148)$ & $\begin{array}{c}146 \\
(141-150)\end{array}$ & $0.02 *$ & & $\begin{array}{c}181 \\
(176-186)\end{array}$ & $180(176-183)$ & $\begin{array}{c}173 \\
(165-180)\end{array}$ & 0.09 & \\
\hline Pantothenic acid & & & & & 0.92 & & & & & $<0.01 *$ \\
\hline Non-exercisers & $2.7(2.7-2.7)$ & $2.7(2.7-2.7)$ & $2.7(2.6-2.7)$ & 0.19 & & $3.1(3.1-3.2)$ & $3.1(3.1-3.2)$ & $3.2(3.1-3.2)$ & 0.08 & \\
\hline Exercisers & $2.9(2.8-2.9)$ & $2.9(2.8-2.9)$ & $2.8(2.8-2.9)$ & 0.21 & & $3.3(3.2-3.4)$ & $3.3(3.2-3.3)$ & $3.2(3.1-3.3)$ & 0.05 & \\
\hline Vitamin C & & & & & 0.99 & & & & & 0.36 \\
\hline Non-exercisers & $41(40-42)$ & $40(39-41)$ & $40(38-41)$ & 0.16 & & $58(55-60)$ & $57(56-58)$ & $58(55-61)$ & 0.87 & \\
\hline Exercisers & $46(45-47)$ & $45(44-46)$ & $45(43-47)$ & 0.20 & & $65(62-67)$ & $62(61-64)$ & $58(54-62)$ & $<0.01 *$ & \\
\hline Sodium & & & & & 0.06 & & & & & 0.07 \\
\hline Non-exercisers & $\begin{array}{c}1896 \\
(1869-1923)\end{array}$ & $\begin{array}{c}1929 \\
(1911-1947)\end{array}$ & $\begin{array}{c}1916 \\
(1882-1950)\end{array}$ & 0.25 & & $\begin{array}{c}2089 \\
(2048-2130)\end{array}$ & $\begin{array}{c}2095 \\
(2071-2119)\end{array}$ & $\begin{array}{c}2101 \\
(2055-2148)\end{array}$ & 0.65 & \\
\hline Exercisers & $\begin{array}{c}1945 \\
(1920-1970)\end{array}$ & $\begin{array}{c}1934 \\
(1916-1952)\end{array}$ & $\begin{array}{c}1905 \\
(1864-1945)\end{array}$ & 0.13 & & $\begin{array}{c}2148 \\
(2105-2190)\end{array}$ & $\begin{array}{c}2156 \\
(2129-2184)\end{array}$ & $\begin{array}{c}2104 \\
(2039-2170)\end{array}$ & 0.48 & \\
\hline Potassium & & & & & 0.73 & & & & & $<0.01^{*}$ \\
\hline Non-exercisers & 943 (928-959) & 931 (921-941) & $\begin{array}{c}922 \\
(903-942)\end{array}$ & 0.09 & & $\begin{array}{c}1151(1124- \\
1177)\end{array}$ & $\begin{array}{c}1149 \\
(1134-1165)\end{array}$ & $\begin{array}{c}1157 \\
(1127-1187)\end{array}$ & 0.76 & \\
\hline Exercisers & $\begin{array}{c}1018 \\
(1003-1033)\end{array}$ & $\begin{array}{c}1004 \\
(993-1015)\end{array}$ & $\begin{array}{c}990 \\
(965-1015)\end{array}$ & 0.05 & & $\begin{array}{c}1251(1223- \\
1279)\end{array}$ & $\begin{array}{c}1231 \\
(1213-1248)\end{array}$ & $\begin{array}{c}1178 \\
(1135-1221)\end{array}$ & $\begin{array}{c}<0.01 \\
*\end{array}$ & \\
\hline Calcium & & & & & 0.27 & & & & & $0.001 *$ \\
\hline Non-exercisers & $176(172-181)$ & $175(172-178)$ & $\begin{array}{c}175 \\
(169-180)\end{array}$ & 0.58 & & $\begin{array}{c}225 \\
(217-232)\end{array}$ & $229(224-233)$ & $\begin{array}{c}230 \\
(222-238)\end{array}$ & 0.32 & \\
\hline Exercisers & $199(194-203)$ & 194 (190-197) & $\begin{array}{c}193 \\
(186-200)\end{array}$ & 0.07 & & $\begin{array}{c}250 \\
(242-258)\end{array}$ & $245(240-250)$ & $\begin{array}{c}242 \\
(229-254)\end{array}$ & 0.20 & \\
\hline Magnesium & & & & & 0.60 & & & & & $0.001^{*}$ \\
\hline Non-exercisers & $107(105-108)$ & $106(105-107)$ & $\begin{array}{c}105 \\
(103-106)\end{array}$ & 0.09 & & $\begin{array}{c}118 \\
(116-120)\end{array}$ & $118(116-119)$ & $\begin{array}{c}119 \\
(117-122)\end{array}$ & 0.29 & \\
\hline Exercisers & $113(111-114)$ & $111(110-112)$ & $\begin{array}{c}110 \\
(108-112)\end{array}$ & $0.03 *$ & & $\begin{array}{c}126 \\
(124-128)\end{array}$ & $125(123-126)$ & $\begin{array}{c}121 \\
(117-124)\end{array}$ & $0.03 *$ & \\
\hline Phosphorus & & & & & 0.37 & & & & & $<0.01 *$ \\
\hline Non-exercisers & $426(421-431)$ & $427(423-430)$ & $\begin{array}{c}424 \\
(418-430)\end{array}$ & 0.80 & & $\begin{array}{c}477 \\
(470-485)\end{array}$ & $482(478-487)$ & $\begin{array}{c}487 \\
(478-495)\end{array}$ & 0.10 & \\
\hline Exercisers & $448(443-453)$ & $446(442-449)$ & $\begin{array}{c}442( \\
434-450)\end{array}$ & 0.16 & & $\begin{array}{c}507 \\
(499-515)\end{array}$ & $501(496-506)$ & $\begin{array}{c}491 \\
(479-503)\end{array}$ & $0.03 *$ & \\
\hline Iron & & & & & 0.24 & & & & & $0.04 *$ \\
\hline Non-exercisers & $3.1(3.0-3.1)$ & $3.1(3.1-3.1)$ & $3.1(3.0-3.1)$ & 0.79 & & $3.7(3.6-3.8)$ & $3.7(3.7-3.8)$ & $3.8(3.7-3.8)$ & 0.35 & \\
\hline Exercisers & $3.3(3.3-3.3)$ & $3.3(3.2-3.3)$ & $3.2(3.2-3.3)$ & 0.16 & & $3.9(3.9-4.0)$ & $3.9(3.8-3.9)$ & $3.8(3.6-3.9)$ & $0.02 *$ & \\
\hline
\end{tabular}


Impact of Interaction of Psychological Stress with Habitual Exercise on Dietary Intake

Table 3 (continued). Interactions between SRPS and Habitual Exercise Level in the Dietary Intake of Energy and Food Types in Middle-aged Japanese men and Women ${ }^{1}$

\begin{tabular}{|c|c|c|c|c|c|c|c|c|c|c|}
\hline \multirow{3}{*}{$\begin{array}{c}\text { (g or mg/1000kcal/ } \\
\text { day) }\end{array}$} & \multicolumn{5}{|c|}{ Men $(n=5,587)$} & \multicolumn{5}{|c|}{ Women $(n=2,718)$} \\
\hline & \multicolumn{3}{|c|}{ SRPS } & \multirow[b]{2}{*}{$\begin{array}{l}\mathrm{p} \text { for } \\
\text { trend }\end{array}$} & \multirow[b]{2}{*}{$\begin{array}{c}\mathrm{p} \text { for } \\
\text { int }\end{array}$} & \multicolumn{3}{|c|}{ SRPS } & \multirow[b]{2}{*}{$\begin{array}{l}\mathrm{p} \text { for } \\
\text { trend }\end{array}$} & \multirow[b]{2}{*}{$\begin{array}{c}\mathrm{p} \text { for } \\
\text { int }\end{array}$} \\
\hline & $\begin{array}{c}\text { Low }^{2} \\
(\mathrm{n}=1,558)\end{array}$ & $\begin{array}{l}\text { Moderate }^{2} \\
(\mathrm{n}=3,247)\end{array}$ & $\begin{array}{c}\text { High }^{2} \\
(\mathrm{n}=782)\end{array}$ & & & $\begin{array}{c}\text { Low }^{2} \\
(\mathrm{n}=641)\end{array}$ & $\begin{array}{l}\text { Moderate }^{2} \\
(\mathrm{n}=1,691)\end{array}$ & $\begin{array}{c}\text { High }^{2} \\
(\mathrm{n}=386)\end{array}$ & & \\
\hline Zinc & & & & & 0.58 & & & & & 0.84 \\
\hline Non-exercisers & $3.6(3.6-3.7)$ & $3.7(3.6-3.7)$ & $3.6(3.6-3.7)$ & 0.86 & & $4.1(4.0-4.1)$ & $4.1(4.1-4.1)$ & $4.1(4.1-4.2)$ & 0.29 & \\
\hline Exercisers & $3.7(3.7-3.7)$ & $3.7(3.7-3.7)$ & $3.7(3.7-3.7)$ & 0.58 & & $4.2(4.1-4.2)$ & $4.1(4.1-4.2)$ & $4.1(4.0-4.2)$ & $0.01 *$ & \\
\hline Copper & & & & & 0.85 & & & & & 0.08 \\
\hline Non-exercisers & $\begin{array}{c}0.56 \\
(0.55-0.56)\end{array}$ & $\begin{array}{c}0.56 \\
(0.55-0.56)\end{array}$ & $\begin{array}{c}0.55 \\
(0.54-0.56)\end{array}$ & $0.04 *$ & & $\begin{array}{c}0.62 \\
(0.61-0.63)\end{array}$ & $\begin{array}{c}0.62(0.61- \\
0.62)\end{array}$ & $\begin{array}{c}0.62 \\
(0.61-0.63)\end{array}$ & 0.33 & \\
\hline Exercisers & $\begin{array}{c}0.58 \\
(0.57-0.58)\end{array}$ & $\begin{array}{c}0.58 \\
(0.57-0.58)\end{array}$ & $\begin{array}{c}0.57 \\
(0.56-0.58)\end{array}$ & 0.12 & & $\begin{array}{c}0.63 \\
(0.62-0.64)\end{array}$ & $\begin{array}{c}0.63(0.63- \\
0.64)\end{array}$ & $\begin{array}{c}0.63 \\
(0.61-0.64)\end{array}$ & 0.50 & \\
\hline Manganese & & & & & 0.84 & & & & & 0.21 \\
\hline Non-exercisers & $\begin{array}{c}1.87 \\
(1.84-1.90)\end{array}$ & $\begin{array}{c}1.88 \\
(1.86-1.90)\end{array}$ & $\begin{array}{c}1.87 \\
(1.83-1.91)\end{array}$ & 0.87 & & $\begin{array}{c}2.07 \\
(2.02-2.11)\end{array}$ & $\begin{array}{c}2.09 \\
(2.06-2.12)\end{array}$ & $\begin{array}{c}2.13 \\
(2.07-2.18)\end{array}$ & 0.07 & \\
\hline Exercisers & $\begin{array}{c}1.90 \\
(1.87-1.93) \\
\end{array}$ & $\begin{array}{c}1.88 \\
(1.86-1.90) \\
\end{array}$ & $\begin{array}{c}1.91 \\
(1.86-1.95) \\
\end{array}$ & 0.81 & & $\begin{array}{c}2.08 \\
(2.04-2.13) \\
\end{array}$ & $\begin{array}{c}2.08 \\
(2.05-2.11) \\
\end{array}$ & $\begin{array}{c}2.09 \\
(2.02-2.16) \\
\end{array}$ & 0.87 & \\
\hline
\end{tabular}

NS, not significant; SRPS, self-reported psychological stress; int, interaction; SFA, Saturated fatty acid; MUFA, Monounsaturated fatty acid, PUFA;polyunsaturated fatty acid; ${ }^{*} \mathrm{p}<0.05 ;{ }^{1}$ Multi-adjusted linear regression analyses were adjusted for age, body mass index, exercise, smoking, energy, alcohol intake, history of disease (hypertension and diabetes mellitus) and sleeping time. Drinking, exercise and history of disease were assumed to be dichotomous variables (non-drinker, non-exercisers or no history of hypertension or diabetes mellitus=0; drinker, habitual exercisers or having a history of hypertension or diabetes mellitus=1). SRPS levels and smoking were assumed to be a trichotomous variable (low or never=0; moderate or past $=1$; high or current $=2$ ). Dietary intake of 58 food items was estimated from a food frequency questionnaire; ${ }^{2}$ Values are shown as the adjusted means (standard error); ${ }^{3}$ Non-exercisers $(\operatorname{men}=2,918$; women $=1,485) ;{ }^{4}$ Exercisers $($ men $=2,669 ;$ women $=1,233)$; akcal $/$ day, bug $/ 1,000 \mathrm{kcal}$

the dietary intake of "cakes, cookies, and biscuits", and "rice crackers, rice cakes, and Japanese-style pancakes" and negatively associated with the dietary intake of "raw vegetables used in salad", green leafy vegetables, "tomatoes, tomato ketchup, boiled tomatoes, and stewed tomatoes", "mayonnaise and salad dressing", and whiskey ( $\mathrm{p}$ for trend $<0.05$ for all). In men, significant interactions were found between SRPS levels and habitual exercise in the intake of "raw vegetables used in salad (cabbage and lettuce)", green leafy vegetables, and "fruit juice and vegetable juice" ( $p$ for interaction $<0.05$ for all). In female non-exercisers, SRPS was positively associated with the dietary intake of green tea and negatively associated with the dietary intake of "radishes and turnips". In female exercisers, SRPS was positively associated with the dietary intake of ice cream and negatively associated with "pork and beef", "ham, sausages, and bacon", canned tuna, and wine. In women, significant interactions were found between SRPS levels and habitual exercise in the intake of "pork and beef", "low fat milk and yogurt", natto (fermented soybean), "carrot and squash", other root vegetables (onions, burdock, and lotus root), mushrooms, seaweeds, and wine ( $\mathrm{p}$ for interaction $<0.05$ for all).

Regarding the dietary intake of nutrients, Table 3 shows the significant interactions between SRPS and habitual exercise levels among middle-aged Japanese men and women. In male non-exercisers, SRPS was positively associated with the dietary intake of saturated fatty acids and negatively associated with the dietary intake of copper. In male exercisers, SRPS was negatively associated with the dietary intake of insoluble and total dietary fiber, retinol equivalent, folate, and magnesium. In men, no significant interactions were found between SRPS levels and habitual exercise in the dietary intake of any nutrients. In female non-exercisers, SRPS was positively associated with vitamin B2. In female exercisers, SRPS was negatively associated with protein, animal protein, vitamins E, B1, B6, and C, potassium, magnesium, phosphorus, iron, and zinc ( $\mathrm{p}$ for interaction $<0.05$ for all). In women, significant interactions were found between SRPS levels and habitual exercise in the intake of vegetable protein, soluble, insoluble, and total dietary fiber, daidzein, genistein, carotene, retinol equivalent, vitamin B2, pantothenic acid, potassium, calcium, magnesium, phosphorus, and iron ( $\mathrm{p}$ for interaction $<0.05$ for all).

\section{Discussion}

In the present study, we found significant interactions between SRPS levels and habitual exercise on the dietary intake of "pork and beef", "low fat milk and yogurt", natto (fermented soybean), "carrot and squash", other root vegetables (onions, burdock, and lotus root), mushrooms, seaweeds, wine, vegetable protein, soluble, insoluble, and total dietary fiber, daidzein, genistein, carotene, retinol equivalent, vitamin B2, pantothenic acid, potassium, calcium, magnesium, phosphorus, and iron in women. In men, we found significant interactions between SRPS levels and habitual exercise on the dietary intake of "raw vegetables used in salad (cabbage and lettuce)", green leafy vegetables, and "fruit juice and vegetable juice".

In the present study, a significant interaction was observed between SRPS and habitual exercise on the dietary intake of 3 vegetables, dietary fibers, soy bean products and isoflavones, 4 vitamins, and 5 minerals in women. This suggests that both SRPS and habitual exercise are possible factors that mediate the dietary intake of food and nutrients in women. It also implies that studies of the dietary intake of food and nutrients should analyze SRPS and habitual exercise concurrently. Consumption of vegetables and fruits and the related nutrients decreases the risk of cancer (World Cancer Research Fund American Institute for Cancer Research, 2007). If both PS and habitual exercise change the dietary intake of vegetables, fruits, and the related vitamins and minerals, the risk of cancer might be modulated by both PS and habitual 
exercise. Elucidating the possible factors that change the dietary intake of food and nutrients is important for cancer prevention, because approximately $30 \%$ of cancer cases are linked to diet (Harvard Center for Cancer Prevention, 1996). This is the first study to examine the interaction between PS and habitual exercise on the dietary intake of food and nutrients. Our study results are useful for investigating the association between diet and cancer risk. Further studies using a prospective design are warranted, as our study was conducted using a cross-sectional design.

In the present study, interactions between SRPS and habitual exercise on the dietary intake of 8 foods and 15 nutrients were found in women. However, interactions between SRPS and habitual exercise were found in the dietary intake of only 3 foods in men. These gender differences could be explained by several factors. The sensitivity to PS differs between men and women (Young and Korszun, 2010). Overall, men are more physically active (Zitouni and Guinhouya, 2012; Peer et al., 2013). The dietary intake of food and nutrients differs among men and women (Iso et al., 2005). Therefore, gender differences in PS, exercise, and diet might be some of the reasons that different results were observed in men and women in the present study. Further studies of other ethnic groups are needed to confirm our results.

Perimenopausal women may have physical symptoms, such as hot flashes and tachycardia, and psychological manifestations, such as depression (Soares, 2013). Genistein, one of the isoflavones, improved depressive symptoms (Atteritano et al., 2014). In the present study, significant interactions between SRPS and habitual exercise on the dietary intake of genistein, daidzein, and natto (fermented soy bean) were found in women. Our results imply that both SRPS and habitual exercise affect the dietary intake of isoflavones and soy bean products. The dietary intake of isoflavones and soy bean products by perimenopausal women should be examined in the context of PS and habitual exercise. However, further studies are needed because this study was conducted using a cross-sectional design.

This study has several limitations. First, we did not determine the type, intensity, frequency, and duration of habitual exercise. However, in a large-scale study, physical activity levels assessed using a single item question could be used to properly classify the subjects (Sternfeld et al., 2000). Second, PS was assessed by self-report. An unhealthy lifestyle, including physical inactivity, drinking, smoking, and being overweight, is associated with PS (Heikkila et al., 2013). Previously, we assessed PS by self-report and verified its association with drinking (Endoh et al., 2015b). Therefore, we believe that our SRPS accurately measures PS. Third, the cause and effect relationship across PS, habitual exercise, and diet was not elucidated because of the study design. Fourth, PS is associated with socioeconomic status (Lorant et al., 2003) but we did not collect this information from the study subjects.

We demonstrated a synergic effect of SRPS and habitual exercise on the dietary intake of food and nutrients related to vegetables which a have protective effect against cancer, especially in women. Our study results are useful for cancer research. Further studies are needed to better understand the role of PS, exercise, and dietary intake in cancer prevention.

\section{Acknowledgements}

The authors declare that they have no conflicts of interest. This study was financially supported by the Global COE Program for the Center of Excellence for Innovation in Human Health Sciences from the Ministry of Education, Science, Sports and Culture of Japan.

\section{References}

Atteritano M, Mazzaferro S, Bitto A, et al (2014). Genistein effects on quality of life and depression symptoms in osteopenic postmenopausal women: a 2-year randomized, double-blind, controlled study. Osteoporos Int, 25, 1123-9.

Chida Y, Hamer M, Wardle J, Steptoe A (2008). Do stress-related psychosocial factors contribute to cancer incidence and survival? Nat Clin Pract Oncol, 5, 466-75.

Endoh K, Kuriki K, Kasezawa N, Tohyama K, Goda T (2015a). Association between smoking status and food and nutrient consumption in Japanese: a large-scale cross-sectional study. Asian Pac J Cancer Prev, 16, 6527-34.

Endoh K, Kuriki K, Kasezawa N, Tohyama K, Goda T (2015b). Interactions between psychological stress and drinking status in relation to diet among middle-aged men and women: a large-scale cross-sectional study in Japan. J Nutr Sci Vitaminol (Tokyo), 61, 64-72.

Harvard Center for Cancer Prevention (1996). Harvard report on cancer prevention. volume 1: causes of human cancer. Cancer Causes Control, 7, S3-59.

Heikkila K, Fransson EI, Nyberg ST, et al (2013). Job strain and health-related lifestyle: findings from an individualparticipant meta-analysis of 118,000 working adults. Am J Public Health, 103, 2090-7.

Iso H, Date C, Noda H, et al (2005). Frequency of food intake and estimated nutrient intake among men and women: the JACC Study. J Epidemiol, 15, 24-42.

Jansson C, Jeding K, Lagergren J (2009). Job strain and risk of esophageal and cardia cancers. Cancer Epidemiol, 33, 473-5.

Kobayashi S, Honda S, Murakami K, et al (2012). Both comprehensive and brief self-administered diet history questionnaires satisfactorily rank nutrient intakes in Japanese adults. J Epidemiol, 22, 151-9.

Kobayashi S, Murakami K, Sasaki S, et al (2011). Comparison of relative validity of food group intakes estimated by comprehensive and brief-type self-administered diet history questionnaires against $16 \mathrm{~d}$ dietary records in Japanese adults. Public Health Nutr, 14, 1200-11.

Laugero KD, Falcon LM, Tucker KL (2011). Relationship between perceived stress and dietary and activity patterns in older adults participating in the Boston Puerto Rican Health Study. Appetite, 56, 194-204.

Lorant V, Deliege D, Eaton W, et al (2003). Socioeconomic inequalities in depression: a meta-analysis. Am J Epidemiol, 157, 98-112.

Massie MJ (2004). Prevalence of depression in patients with cancer. J Natl Cancer Inst Monogr, 2004, 57-71.

Mochizuki K, Miyauchi R, Misaki Y, et al (2011). Accumulation of visceral fat is positively associated with serum ALT and gamma-GTP activities in healthy and preclinical middleaged Japanese men. J Nutr Sci Vitaminol (Tokyo), 57, 65-73.

Peer N, Bradshaw D, Laubscher R, Steyn N, Steyn K (2013). Urban-rural and gender differences in tobacco and alcohol 
use, diet and physical activity among young black South Africans between 1998 and 2003. Glob Health Action, 6, 19216.

Perales F, Pozo-Cruz JD, Pozo-Cruz BD (2014). Impact of physical activity on psychological distress: a prospective analysis of an Australian national sample. Am J Public Health, 104, 91-7.

Santos MC, Horta BL, Amaral JJ, et al (2009). Association between stress and breast cancer in women: a meta-analysis. Cad Saude Publica, 25, 453-63.

Schernhammer ES, Hankinson SE, Rosner B, et al (2004). Job stress and breast cancer risk: the nurses' health study. Am J Epidemiol, 160, 1079-86.

Soares CN (2013). Depression in peri- and postmenopausal women: prevalence, pathophysiology and pharmacological management. Drugs Aging, 30, 677-85.

Sternfeld B, Cauley J, Harlow S, Liu G, Lee M (2000). Assessment of physical activity with a single global question in a large, multiethnic sample of midlife women. Am J Epidemiol, 152, 678-87.

World Cancer Research Fund and American Institute for Cancer Research (2007). Food, nutrition, physical activity, and the prevention of cancer: a global perspective

World Health Organization (2015). Cancer factsheet No.297

Young E, Korszun A (2010). Sex, trauma, stress hormones and depression. Mol Psychiatry, 15, 23-8.

Zitouni D, Guinhouya BC (2012). Maturity negates the genderrelated difference in physical activity among youth. Is this equally justified whatever the accelerometer cut-off point used? J Sci Med Sport, 15, 327-33. 